\title{
Up-regulation of FN1, Activation of Maturation Promoting Factor and Associated Signaling Pathway Facilitates Epithelial-Mesenchymal Transition, Inhibits Apoptosis and Elevates Proliferation Rate of Breast Cancer Cells: In Silico Analysis of Microarray Datasets
}

Yasir Hameed

The Islamia University of Bahawalpur Pakistan

Samina Ejaz ( $\nabla$ saminaejazsyed@yahoo.com)

The Islamia University of Bahawalpur Pakistan

\section{Research}

Keywords: Breast cancer, GO analysis, KEGG pathway analysis, Hub genes, Cytoscape software

Posted Date: September 8th, 2020

DOI: https://doi.org/10.21203/rs.3.rs-71394/v1

License: (c) (i) This work is licensed under a Creative Commons Attribution 4.0 International License.

Read Full License 


\section{Abstract}

Background: Knowing that the molecular mechanisms underlying breast cancer $(\mathrm{BC})$ are not yet fully understood it was considered worth to launch investigation for the detection of key molecular pathways and associated genes and proteins.

Methods: In total two microarray based datasets (GSE10810 and GSE29431), consisting of 89 breast cancer samples and 31 controls, were retrieved from the Gene Expression Omnibus (GEO) database and processed to identify the differentially expressed genes (DEGs). The pathway and functional enrichment analyses of DEGs were performed using DAVID online tool. Protein-Protein interaction (PPI) network was constructed using an online tool STRING and visualized through Cytoscape software to identify the significant module and hub genes via MCODE and Cytohubba applications. The identified hub genes were then further analyzed to document their response in Kaplan-Meier (KM) survival curve analysis, investigate differential expression profile and its correlation with promoter's methylation status, and finally for the construction of the gene-drug interaction network.

Results: In total 449 DEGs were detected including 151 up-regulated and 298 down-regulated genes. The identified DEGs were enriched in various cancer related biological functions and pathways. Based on PPI network analysis of the DEGs, six hub genes, CDK1, FN1, AURKA, CCNB2, BIRC5, and TOP2A, were correlated with worse overall survival (OS) of the breast cancer patients.

Conclusion: The extensive in silico analysis has been helpful to evaluate evidence highlighting the prominent role played by the identified hub genes in stimulating breast tumor growth through the activation of Maturation Promoting Factor (MPF), PI3K/AKT signaling, and associated pathways that could be targeted for devising effective treatment strategies.

\section{Background}

Breast cancer $(\mathrm{BC})$ is the most common type of non-cutaneous malignancy among female with a high recurrence rate. The global burden of breast cancer has been enhanced significantly in recent years and it is estimated that more than 1.7 million individuals are being diagnosed with breast cancer annually, worldwide, and approximately 521,000 individuals succumb to the disease [1]. Reproductive and dietary changes are contributing to the increased morbidity of breast cancer. However, the precise molecular mechanisms underlying breast carcinoma are still uncertain. The division of breast cancer into different subtypes is based on the difference of molecular characteristics, clinical outcomes and histopathological appearance. Based on the molecular characteristics breast cancer is categorized into six different subtypes: normal-like, luminal A and B, HER2-positive, basal-like, and claudin-low. The basal-like and claudin-low subtypes are recognized by the lack of estrogen receptor (ER) and progesterone receptor (PR), respectively. The lack of HER2 expression, belongs to triple-negative breast cancer (TNBC) which accounts for $10-20 \%$ of all breast cancer cases [2]. 
Nowadays 3 different biomarkers including the estrogen receptor (ER), progesterone receptor (PR) and human epidermal growth factor (EGF) receptor 2 (HER2) are being exploited for diagnosis, prognosis and the treatment of breast cancer [3]. However, no biomarker exists to date which can be employed for the early diagnosis and treatment of breast carcinoma [4]. Therefore, it is necessary to explore the molecular regulatory pathways to understand biology of breast carcinoma and identify molecular markers / targets that can be used in future as tools for early diagnosis and monitoring of the disease.

The role of microarray analysis in studying the genetic variability is well established in oncology research. In recent years, DNA microarray analysis technique has been emerged as a rapid, high-throughput detection technology due to its potential to simultaneously monitor the differential expression of various genes or miRNAs in diseased and normal samples $[5,6]$. The microarray data of breast cancer patients and normal individuals have been stored in online electronic repositories such as GEO [7-9]. Therefore, in present work, we identified differentially expressed genes (DEGs) by analyzing two breast cancer mRNA microarray datasets (GSE10810 and GSE61304). We then aimed to perform gene ontology (GO) and Kyoto Encyclopedia of Genes and Genomes (KEGG) pathway analysis of GEGs, construction of proteinprotein interaction network (PPI) for significant module and hub genes identification. We hypothesized that the pathway enrichment analysis and further validation of Hub genes participation in tumor biology through in-silico analysis of available wet laboratories' data would be helpful for understanding the molecular mechanism underlying breast cancer. The information thus generated might also provide new insights for the identification of novel breast cancer biomarkers.

\section{Material And Methods}

\section{Microarray data sets retrieval}

The Gene Omnibus (GEO) (available at: https://www.ncbi.nlm.nih.gov/gds), a free international public repository of genomics data including microarray and next-generation sequencing (NGS) data, was accessed to retrieve the microarray datasets. In the present study, we retrieved two microarray datasets (GSE10810 and GSE61304) which contained microarray expression profile data using the Affymetrix U133 Plus 2.0 gene expression array from GEO. The data set GSE10810 consisted of total 58 samples including 27 normal and 31 breast cancer samples. While GSE61304 data set comprised of 62 sample including 4 normal and 58 breast cancer samples.

\section{Identification of differentially expressed genes (DEGs)}

GSE10810 and GSE61304 data sets were selected and analyzed to identify differentially expressed genes (DEGs) using software GEO2R (available at: https://www.ncbi.nlm.nih.gov/geo/geo2r/). In the present study, all the top 250 genes with fold change $\geq 1.5$ and $p<0.05$ were considered as DEGs.

\section{Functional and pathway enrichment analysis}


Gene Ontology (GO) analysis and the Kyoto Encyclopedia of Genes and Genomes (KEGG) pathway enrichment analysis of DEGs were conducted using Database for Annotation, Visualization and Integration Discovery, DAVID version 6.8 [10]. A P value $<0.05$ was chosen as the cut-off criterion. GO is a set of coherent terminology to describe the biological processes (BP). While KEGG analysis was performed to understand the signaling pathways involving DEGs.

\section{PPI network construction, module and KEGG pathway analysis}

The online tool STRING (available at: http://string.embl.de/) is a biological repository, designed to construct the PPI network of DEGs based on the known and predicted PPIs, and then analyze the functional interactions between proteins [11]. Using STRING, a PPI network of all the DEGs was constructed with a confidence score $\geq 0.7$. Subsequently, the PPI network was then visualized using Cytoscape software (version 3.5.1). Furthermore, the plug-in of Molecular Complex Detection (MCODE) in Cytoscape software (Bader and Hogue, 2003) was applied to explore the significant module containing hub genes in the constructed PPI network. The advanced options were set as degree cutoff $=2, \mathrm{~K}$-Core $=$ 2, and Node Score Cutoff $=0.2$ for module identification and then top 6 DEGs with highest degree of centrality were also found using Cytohubba application through Cytoscape software. Subsequently, the KEGG pathway enrichment analysis of the identified hub genes was conducted using Database for Annotation, Visualization and Integration Discovery (DAVID) version 6.8 [10]. The P value $<0.05$ was selected as the cut-off criterion.

\section{Survival analysis of hub genes}

The Kaplan-Meier plotter (available at: http://kmplot.com/analysis/) could determine the impact of 54,675 genes on survival duration using 18,674 cancer samples [12]. The purpose of the tool is biomarker based meta-analysis to provide an advantage in clinical decisions, health care policies, and resource allocation [13]. In present study, we analyzed effect of each identified hub gene on the overall survival of patients through the Kaplan-Meier plotter. Patients were divided into two classes according to the median of each hub gene expression in Kaplan-Meier plotter for overall survival. This classification method could display the survival probability differences between high-expression class and lowexpression class.

\section{Validation of hub gene expression and their promoters' methylation analysis}

The relative transcription and translation expression and methylation analysis of the six hub genes was performed using online platform, UALCAN (available at; http://ualcan.path.uab.edu), a user-friendly web 
resource for analyzing cancer transcriptome data i.e. TCGA and MET500 transcriptome sequencing [14]. UALCAN allows users to perform in silico validation of potential genes of interest. One of the user-friendly features of this platform is that it allows user to perform the analysis of relative expression and promoter's methylation status of a query gene(s) across tumor and normal samples, as well as in subgroups based upon various clinicopathological features such as individual age, gender, tumor stages etc. The database uses quartile ranges from minimum to maximum values that indicate differential expression i.e., ranging from lower to higher, respectively. Expression of transcripts was measured as number of transcripts per million (TPM). UALCAN web portal integrates mass spectrometry-based proteomic data from The Clinical Proteomic Tumor Analysis Consortium (CPTAC) in various cancer types. To have an account of hub genes protein's expression we carried out CPTAC analysis. Z-value was used to measure the standard deviations from the median documented across samples of a particular group. UALCAN also links the gene expression with methylation status either hypo or hyper based on beta values i.e., 0 for fully unmethylated state and 1 for fully methylated state. The database uses cut-off values for hypo-methylation (0.3-0.2) and hyper-methylation (0.7-0.5). Therefore, we explored the relative expression and corresponding gene's promoter methylation status of six hub genes with the help of UALCAN based on various tumor molecular subtypes and clinicopathological features of breast cancer.

\section{Hub gene-drug interaction network analysis}

Comparative Toxicogenomics Database (CTD) was utilized to construct the hub gene-drug interaction for chemotherapeutic drugs that have the potential to reduce or enhance the mRNA or protein expression levels of the hub genes [15]. Briefly, six hub genes including CDK1, FN1, AURKA, CCNB2, BIRC5, and TOP2A were searched in CTD database, and the hub gene-drug interaction networks were constructed using Gliffy online tool (available at: https://www.gliffy.com/examples/network-diagrams).

\section{Results}

From both data sets (GSE10810 and GSE61304) a total 250 significantly differential expressed genes, either up- or down-regulated, were identified for each dataset. In data set GSE10810 out of 228 genes 37 genes were up-regulated and 191 were down-regulated genes. While in dataset GSE61304 out of total 221 DEGs 114 genes were up-regulated and 107 genes were down-regulated regulated including 33 common up-regulated / down-regulated genes. All the DEGs were clustered in the form of one table. Hence, a total of 418 genes were listed as DEGs that expressed a fold change of value $\geq 1.5$ or $\leq-1.5$ and $p<0.05$.

\section{GO term enrichment analysis of DEGs}

For GO and KEGG pathway analysis, the identified significant DEGs were submitted to the online software DAVID. The results of the GO analysis showed that up-regulated DEGs were significantly enriched in biological processes like 'cell division', 'nuclear division', 'organelle fission', 'mitotic cell cycle process', and 'mitotic nuclear division' (Figure 1A; Table 1). The down-regulated DEGs were mainly enriched in 'Lipid 
localization', 'muscle system processes, 'muscle organ development' and 'single-organ metabolic process (Figure 1B; Table 2). 
Table 1

Gene ontology (GO) analysis of up-regulated DEGs associated with breast cancer

\section{Gene Name}

KIF4A, NEK2, AURKA, CEP55, PTTG1, KIF2C, SPC25 CCNA2, TOP2A, ASPM, KIF14, CDK1, NUF2, TPX2, NUSAP1, CENPF, BIRC5, UBE2C, CDK5, PPP1CA, CCNB2, KNL1, ZWINT, CKS2, KIF20A

KIF14, CDK1, KIF4A, MKI67, NEK2, PTTG3P, TPX2, NUF2, NUSAP1, CENPF, BIRC5, AURKA, PTTG1, CEP55, UBE2C, SPC25, KIF2C, CCNB2, KNL1, ZWINT, CKS2, TOP2A, CCNA2, ASPM

KIF14, CDK1, KIF4A, MKI67, NEK2, PTTG3P, TPX2, NUF2, NUSAP1, CENPF, BIRC5, AURKA, PTTG1, CEP55, UBE2C, SPC25, KIF2C, CCNB2, KNL1, ZWINT, CKS2, TOP2A, CCNA2, ASPM

KIF4A, NEK2, AURKA, PTTG1, CEP55, GTSE1, KIF2C, SPC25, FANCI, TOP2A, CCNA2, ASPM, KIF14, CDK1, TPX2, NUF2, NUSAP1, CENPF, BIRC5, UBE2C, INHBA, CCNB2, KNL1, ZWINT, RRM2, CKS2, KIF20A

KIF14, CDK1, KIF4A, NEK2, NUF2, TPX2, NUSAP1, CENPF, BIRC5, AURKA, PTTG1, CEP55, UBE2C, KIF2C, SPC25, CCNB2, KNL1, ZWINT, CCNA2, ASPM

KIF4A, NEK2, AURKA, PTTG1, CEP55, GTSE1, SPC25, KIF2C, FANCI, TOP2A, CCNA2, ASPM, KIF14, CDK1, MKI67, DTL, PTTG3P, TPX2, NUF2, CENPF, TBRG4, NUSAP1, BIRC5, UBE2C, CDK5, INHBA, CCNB2, KNL1, ZWINT, RRM2, CKS2, KIF20A

KIF14, KIF4A, NEK2, PTTG3P, NUF2, NUSAP1, CENPF BIRC5, PTTG1, CEP55, UBE2C, KIF2C, SPC25, HJURP, KNL1, ZWINT, TOP2A

KIF14, KIF4A, NEK2, NUF2, NUSAP1, CENPF, BIRC5, CEP55, UBE2C, SPC25, KIF2C, KNL1, ZWINT, TOP2A

CTHRC1, HIST2H2AA4, U2AF2, AP2S1, AURKA, PTTG1, KIF2C, SAP30, WISP1, HPSE, TOP2A, CCNA2, COL11A1, ASPM, COL10A1, KIF14, CDK1, TPX2, NUSAP1, UBE2C, CDK5, TRPM2, ZNF335, MMP11, ADRM1, INHBA, PPP1CA, UHRF1, ZWINT, RRM2, CFL1, MFAP2, IBSP, KIF4A, SYNDIG1, NEK2, KIAA0101, CEP55, ATP6V0B, TPM3, TK1, SPC25, PFN1, SLC11A1, HJURP, COMP, FN1, MKI67, PTTG3P, NUF2, CENPF, BIRC5, STAT1, GJB2, POC1A, CCNB2, ARF1, KNL1, SULF1, CKS2, SH3BGRL3, HIST1H2AM, PARP1, KIF20A

KIF14, CDK1, KIF4A, HIST2H2AA4, MKI67, NEK2, PTTG3P, NUF2, CENPF, NUSAP1, BIRC5, AURKA,
Gene term/Function

GO:0051301 cell division

GO:0000280 nuclear

division

$24<$

0.05

$\begin{array}{ll}\text { Gene } & \text { P- } \\ \text { Count } & \text { value }\end{array}$

25

$<$ 0.05
G0:0048285 organelle fission
$<$ 0.05
G0:1903047 mitotic cell cycle process
27

$<$ 0.05
G0:0007067 mitotic nuclear division

G0:0022402 cell cycle process
32

20 0.05
G0:0007059 chromosome segregation

G0:0000819 sister chromatid segregation

GO:0016043 cellular component organization
17

$<$ 0.05

14

64
$<$ 0.05 
CEP55, PTTG1, UBE2C, ZNF335, SPC25, KIF2C,

SAP30, UHRF1, KNL1, HJURP, ZWINT, PARP1,

HIST1H2AM, TOP2A

CTHRC1, HIST2H2AA4, U2AF2, AP2S1, AURKA,

PTTG1, KIF2C, SAP30, WISP1, HPSE, TOP2A,

CCNA2, COL11A1, ASPM, COL10A1, KIF14, CDK1,

TPX2, NUSAP1, UBE2C, CDK5, TRPM2, ZNF335,

MMP11, ADRM1, INHBA, PPP1CA, UHRF1, ZWINT,

RRM2, CFL1, MFAP2, IBSP, KIF4A, SYNDIG1, NEK2,

KIAA0101, CEP55, ATP6V0B, TPM3, TK1, SPC25,

PFN1, SLC11A1, HJURP, COMP, FN1, MKI67, PTTG3P,

NUF2, CENPF, BIRC5, STAT1, GJB2, POC1A, CCNB2,

ARF1, KNL1, SULF1, CKS2, SH3BGRL3, HIST1H2AM,

PARP1, KIF20A

KIF14, CDK1, MKI67, DTL, NEK2, KIAA0101, TPX2, NUSAP1, TBRG4, CENPF, BIRC5, AURKA, UBE2C,

CDK5, GTSE1, INHBA, CCNB2, FANCI, ZWINT, CKS2,

TOP2A, CCNA2, ASPM

KIF14, CDK1, MKI67, NEK2, TPX2, NUSAP1, CENPF, BIRC5, AURKA, UBE2C, GTSE1, FANCI, ZWINT, CKS2, CCNA2, TOP2A

CDK1, DTL, FANCI, KNL1, ZWINT, CENPF, AURKA, BIRC5, CCNA2, TOP2A, GTSE1

KIF14, CDK1, KIF4A, NEK2, NUF2, KIAA0101, TPX2, CENPF, NUSAP1, BIRC5, AURKA, CEP55, UBE2C, CDK5, TRPM2, TPM3, SPC25, KIF2C, PFN1, ARF1, KNL1, ZWINT, CFL1, SH3BGRL3, TOP2A, ASPM, KIF20A

KIF14, CDK1, NEK2, TPX2, CENPF, BIRC5, AURKA, UBE2C, GTSE1, INHBA, CCNB2, FANCI, RRM2, CKS2, CCNA2

KIF14, KIF2C, KIF4A, NEK2, ZWINT, NUSAP1, CENPF, CEP55, UBE2C

KIF4A, HIST2H2AA4, NEK2, U2AF2, KIAA0101, AURKA, CEP55, PTTG1, ATP6V0B, TPM3, SPC25, PFN1, KIF2C, SAP30, HJURP, TOP2A, CCNA2, ASPM, KIF14, CDK1, MKI67, PTTG3P, NUF2, TPX2, CENPF, NUSAP1, BIRC5, UBE2C, CDK5, ZNF335, TRPM2, UHRF1, CCNB2, ARF1, KNL1, ZWINT, CFL1, CKS2, SH3BGRL3, PARP1, HIST1H2AM, KIF20A

KIF14, CDK1, KIF2C, KIF4A, NEK2, KIAA0101, TPX2, NUSAP1, AURKA, BIRC5, ASPM, KIF20A

CDK1, TNFSF4, MKI67, DTL, NEK2, KIAA0101, S100A11, CENPF, PTTG1, TK1, PFN1, UHRF1, FANCI,
GO:0071840 biogenesis

64

$<$

0.05

$\begin{array}{lll}\text { GO:0051726 regulation of } & 23 & < \\ \text { cell cycle } & & 0.05\end{array}$

GO:0007346 regulation of 16 mitotic cell cycle 0.05

GO:0000075 cell cycle checkpoint

$11<$ 0.05

GO:1902589 singleorganism organelle $27<$ organization
G0:0044772 mitotic cell cycle phase transition
$15<$ 0.05
G0:0000070 mitotic sister 9 chromatid segregation

9

$<$ 0.05

G0:0006996 organelle

organization
$<$

0.05
GO:0000226 microtubule cytoskeleton organization

G0:0006259 DNA

metabolic process
12

$<$ 0.05 
Table 2

Table 2: Gene ontology (GO) analysis of down-regulated DEGs associated with breast cancer

\section{Gene name}

ABCA8, RBP4, CAV1, ABCA9, PPARG, STAT5B, CIDEA ABCB1, ACACB, FZD4, ABCA6, ACVR1C, ABCA5, LEP,

SPX, PLA2R1, GPIHBP1, ANO6
CFLAR, CAV1, SCN3B, CRYAB, PPP1R12B, UTRN, OXTR, KLF15, ATP1A2, KCNIP2, LEP, EDNRB, HSPB6, SORBS1, DMD, MYH11, SCN4B, CNN1, TMOD1

HLF, RBP4, FGFR1, CAV2, CFLAR, CAV1, KCNAB1, CRYAB, FHL1, MRAS, UTRN, SMYD1, FOXP1, TAGLN, S100B, DMD, CFL2, ITGA7

HLF, RBP4, FGFR1, CAV2, CFLAR, CAV1, KCNAB1, CRYAB, FHL1, MRAS, UTRN, SMYD1, TCF7L2, FOXP1, EDNRB, S100B, TAGLN, DMD, CFL2, ITGA7, MYH11,

TMOD 1

ALDH1L1, ZAK, KCNAB1, PDGFA, STAT5B, PPARG, ADH1C, TMEM237, PDE3B, ADH1B, ACVR1C, SPRY2, EDNRB, ASPA, GPC3, PAK3, PPP1R1A, PAK5, LOXL4, PDGFD, FGF1, GHR, NMNAT2, AIFM2, FAXDC2, CRYAB, RDH5, GNAL, MARC1, CLIC5, CA4, HAS3, GPAM, UGP2, ADAMTS5, AOC3, CAV2, RBP4, FGFR1, CAV1, MRAP, ADCYAP1R1, AKAP12, MME, SNX1, OXTR, SLC19A3, CX3CL1, PLPP3, SORBS1, COL6A6, SH3GLB1, FMO2, DMD, PER1, GPD1, BHMT2, KLB, MAOA, NPR1, CIDEA, GYG2, ACACB, ATP1A2, FZD4, SOD3, PCK1, LEP, GLYAT, ATXN3, PDE2A, NTRK2, ALDH2, SPTBN1, HRASLS5, GRK3, ACSM5

RHOJ, CFLAR, CAV1, SCN3B, PDGFA, MRAS, PDLIM3, ATP1A2, FOXP1, KANK1, SEMA5A, CORO2B, ATXN3, SORBS1, PAK3, DMD, CFL2, MYH11, SPTBN1, SCN4B, CNN1, PARVA, TMOD1

ABCA8, RBP4, CAV1, ABCA9, PPARG, ABCB1, ACACB, FZD4, ABCA6, ABCA5, LEP, SPX, PLA2R1, GPIHBP1, ANO6

RHOJ, FGFR1, RBP4, CAV1, PDGFA, KCNAB1, PPARG, PDLIM3, PDE3B, OXTR, CX3CL1, TCF7L2, PLPP3, SEMA5A, GPC3, TMEM100, PDGFD, FGF1, NPR1, SMYD1, ACACB, MCAM, FZD4, FOXP1, LEP, NTRK2, MYH11, PARVA

RHOJ, FGFR1, RBP4, CAV1, PDGFA, KCNAB1, PPARG, PDLIM3, PDE3B, OXTR, CX3CL1, TCF7L2, PLPP3, SEMA5A, GPC3, TMEM100, PDGFD, FGF1, NPR1, SMYD1, ACACB, MCAM, FZD4, FOXP1, LEP, NTRK2, MYH11, PARVA
GO:0030029 actin

filament-based process

GO:0044710 singleorganism metabolic

77

$<$ process

\section{Gene term/function}

G0:0010876 lipid

localization

G0:0003012 muscle

system process

19

Gene

count

18

$<$

0.05
$<$

0.05
$<$ 0.05 value

value

GO:0007517 muscle

G0:0061061 muscle structure development

22

$<$ 0.05

\section{5}

18 
CACHD1, SCN3B, PDGFA, KCNAB1, TLN2, STAT5B, UTRN, PPARG, ITSN1, KCNIP2, KANK1, ACVR1C, SPRY2, EDNRB, SRPX, GPC3, SPX, CFD, GPIHBP1, ANO6, GHR, CLCA4, NUP88, STXBP1, CLIC5, CD300LG, CA4, RELN, GPAM, PROS1, ABCA8, TF, RBP4, FGFR1, CAV1, ABCA9, FHL1, ADCYAP1R1, SNX1, OXTR, SLC19A3, TCF7L2, ABCA6, ABCA5, SEMA5A, SORBS1, SH3GLB1, DMD, PER1, PLA2R1, NPR1, CIDEA, ABCB1, ATP1A2, ACACB, KLF15, FZD4, FOXP1, LEP, SYNE3, PDE2A, SLC16A7, FBLN5, ANXA11, NTRK2, SCN4B, SPTBN1, GRK3, SCARA5

PDGFA, STAT5B, PPARG, PDE3B, KCNIP2, KANK1, ACVR1C, SPRY2, EDNRB, GPC3, SPX, SAA2, PAK3, SEMA3G, ZNF106, TMEM100, PDGFD, FGF1, SIK2, GPIHBP1, ANO6, PPP1R14A, GHR, CRYAB, LIFR, SLIT3, MARC1, HSPB7, TFPI, HSPB2, RELN, GPAM, CHL1, AOC3, PARVA, ABCA8, TF, CAV2, RBP4, FGFR1, CAV1, GNAI1, ADCYAP1R1, SOX5, MME, OXTR, TIMP4, CX3CL1, TCF7L2, SEMA5A, SORBS1, FM02, PER1, PLA2R1, GPD1, KLB, CIDEA, ABCB1, ACACB, KLF15, ATP1A2, FZD4, SOD3, FOXP1, PCK1, LEP, CDKN1C, GLYAT, ATXN3, PDE2A, S100B, FBLN5, ANXA11, NTRK2, SPTBN1, OR10D3

ZAK, KCNAB1, PDGFA, SCN3B, PPARG, UTRN, EIF5, TCEAL7, KCNIP2, ITSN1, ACVR1C, EDNRB, SPRY2, GPC3, ARHGAP20, CDKN2C, PAK3, PPP1R1A, ITIH5, PDGFD, FGF1, GPIHBP1, PPP1R14A, GHR, CRYAB, LDB2, PCOLCE2, GNAL, TFPI, HSPB2, RELN, PROS1, CCDC8, FGFR1, CAV2, CAV1, FHL1, ADCYAP1R1, PPP1R12B, TIMP4, DENND2A, CX3CL1, TCF7L2, FAM13A, PLPP3, SH3GLB1, DMD, PLA2R1, CFLAR, KLB, ACACB, ATP1A2, FZD4, LEP, CDKN1C, NTRK2, SPTBN1, SCN4B

RBP4, FGFR1, CFLAR, CRYAB, FHL1, IGFBP6, PPARG, STAT5B, NPR1, ACACB, DCAF1, FOXP1, SLIT3, SEMA5A, LEP, CDKN1C, SPRY2, FAM107A, GPC3, CDKN2C, SEMA3G, FBLN5, PAK5, FGF1, GPAM, EMP1, GHR

FGFR1, RBP4, CAV1, SCN3B, KCNAB1, PPP1R12B, UTRN, PPARG, SOBP, MME, OXTR, KCNIP2, EDNRB, SPRY2, SPX, SORBS1, PDE1C, DMD, PAK5, CNN1, TMEM100, CFLAR, CRYAB, NPR1, KLF15, ATP1A2, MCAM, FZD4, RDH5, CCDC50, LEP, GNAL, PDE2A, S100B, HSPB6, CLIC5, HSPB7, NTRK2, MYH11, CELF2, SCN4B, OR10D3, RELN, CHL1, TMOD1
RHOJ, ZAK, PDGFA, EZH1, TMEM237, SOBP, PDE3B, KANK1, SPRY2, GPC3, SEMA3G, PAK3, TMEM100, FGF1, GHR, TBX15, CRYAB, STXBP1, SLIT3, CLIC5, CFL2, RELN, ADAMTS5, CHL1, PARVA, FGFR1, RBP4, CAV1, FHL1, SNX1, PALMD, CX3CL1, TCF7L2, PLPP3, SEMA5A, DMD, PALM2, CFLAR, NPR1, MCAM, FZD4, FOXP1, SYNE3, LEP, CDKN1C, LYVE1, S100B, FREM1, NTRK2, ITGA7, MYH11, SPTBN1, SH3D19, TMOD1
G0:1902578 single-

organism localization

69

$<$ 0.05

$\begin{array}{lll}\text { GO:0065009 regulation } & 58 & < \\ \text { of molecular function } & & 0.05\end{array}$

GO:0040007 growth

27 0.05
$<$ 0.05

$\begin{array}{ll}76< & < \\ 0.05\end{array}$

$\begin{array}{lll}\begin{array}{l}\text { GO:0003008 system } \\ \text { process }\end{array} & 45 & < \\ & & 0.05\end{array}$

$\begin{array}{lll}\text { G0:0009653 anatomical } & 54 & < \\ \text { structure morphogenesis } & & 0.05\end{array}$




\begin{tabular}{|c|c|c|c|}
\hline LEP, CAV1, STAT5B, PPARG, CIDEA, ACACB, ACVR1C & G0:0019915 lipid storage & 7 & $\begin{array}{l}< \\
0.05\end{array}$ \\
\hline $\begin{array}{l}\text { CAV2, CAV1, GNAI1, PPARG, STAT5B, PDE3B, OXTR, } \\
\text { TIMP4, KLF15, KANK1, PCK1, ACVR1C, LEP, SORBS1, } \\
\text { ZNF106, SIK2, GHR }\end{array}$ & $\begin{array}{l}\text { GO:1901652 response to } \\
\text { peptide }\end{array}$ & 17 & $\dot{0.05}$ \\
\hline $\begin{array}{l}\text { RHOJ, ZAK, PDGFA, TLN2, MRAS, PDLIM3, KANK1, } \\
\text { SEMA5A, CORO2B, SPRY2, SORBS1, PAK3, DMD, } \\
\text { STARD9, PAK5, CNN1, CFLAR, CRYAB, FOXP1, } \\
\text { SYNE3, ATXN3, CFL2, CEP68, MYH11, SPTBN1, } \\
\text { SH3D19, MAP7D3, TMOD1, CCDC8, PARVA }\end{array}$ & $\begin{array}{l}\text { Go:0007010 cytoskeleton } \\
\text { organization }\end{array}$ & 30 & $\begin{array}{l}< \\
0.05\end{array}$ \\
\hline $\begin{array}{l}\text { CAV2, CAV1, GNAI1, PPARG, STAT5B, PDE3B, OXTR, } \\
\text { TIMP4, KANK1, PCK1, ACVR1C, LEP, SORBS1, } \\
\text { ZNF106, SIK2, GHR }\end{array}$ & $\begin{array}{l}\text { GO:0043434 response to } \\
\text { peptide hormone }\end{array}$ & 16 & $\begin{array}{l}< \\
0.05\end{array}$ \\
\hline $\begin{array}{l}\text { RBP4, FGFR1, CRYAB, FHL1, IGFBP6, PPARG, } \\
\text { STAT5B, NPR1, ACACB, DCAF1, FOXP1, SLIT3, } \\
\text { SEMA5A, LEP, GPC3, FAM107A, CDKN2C, SEMA3G, } \\
\text { FBLN5, GPAM, GHR }\end{array}$ & $\begin{array}{l}\text { GO:0040008 regulation } \\
\text { of growth }\end{array}$ & 21 & $\begin{array}{l}< \\
0.05\end{array}$ \\
\hline
\end{tabular}

\section{KEGG pathway analysis of DEGs}

KEGG pathway analysis of up-regulated DEGs reveled their involvement in various pathways including 'oocyte meiosis', 'p53 signaling pathway', 'focal adhesion' and 'ECM-receptor interaction' (Figure 2A; Table 3). While the KEGG pathway analysis of the down-regulated DEGs revealed their enrichment in 'focal adhesion', 'Rennin secretion', 'regulation of active cytoskeleton', 'ABC transporters' and 'axon guidance' (Figure 2B; Table 4) 
Table 3

Kyoto encyclopedia of genes and genomes pathway analysis of up-regulated DEGs related with breast cancer

\begin{tabular}{|c|c|c|c|c|}
\hline $\begin{array}{l}\text { Pathway } \\
\text { ID }\end{array}$ & Pathway Name & $\begin{array}{l}\text { Gene } \\
\text { count }\end{array}$ & $\begin{array}{l}\mathrm{P}- \\
\text { value }\end{array}$ & Gene name \\
\hline 04114 & Oocyte meiosis & 5 & $\begin{array}{l}< \\
0.05\end{array}$ & $\begin{array}{l}\text { CDK1, PPP1CA, CCNB2, AURKA, } \\
\text { PTTG1 }\end{array}$ \\
\hline 04115 & p53 signaling pathway & 4 & $\begin{array}{l}< \\
0.05\end{array}$ & CDK1, CCNB2, RRM2, GTSE1 \\
\hline 04510 & Focal adhesion & 6 & $\begin{array}{l}<.05 \\
0.05\end{array}$ & $\begin{array}{l}\text { IBSP, PPP1CA, COMP, COL11A1, } \\
\text { FN1, PIK3R2 }\end{array}$ \\
\hline 04512 & ECM-receptor interaction & 4 & $\begin{array}{l}<.05 \\
0.05\end{array}$ & IBSP, COMP, COL11A1, FN1 \\
\hline 04914 & $\begin{array}{l}\text { Progesterone-mediated oocyte } \\
\text { maturation }\end{array}$ & 4 & $\begin{array}{l}< \\
0.05\end{array}$ & CDK1, CCNB2, CCNA2, PIK3R2 \\
\hline 04110 & Cell cycle & 4 & $\begin{array}{l}< \\
0.05\end{array}$ & CDK1, CCNB2, PTTG1, CCNA \\
\hline 05161 & Hepatitis B & 4 & $\dot{0.05}$ & BIRC5, STAT1, CCNA2, PIK3R2 \\
\hline
\end{tabular}


Table 4

Kyoto encyclopedia of genes and genomes pathway analysis of down-regulated DEGs related with breast cancer

\begin{tabular}{|c|c|c|c|c|}
\hline $\begin{array}{l}\text { Pathway } \\
\text { ID }\end{array}$ & Pathway Name & $\begin{array}{l}\text { Gene } \\
\text { count }\end{array}$ & $\begin{array}{l}\mathrm{P}- \\
\text { value }\end{array}$ & Gene name \\
\hline 04510 & Focal adhesion & 12 & $<.05$ & $\begin{array}{l}\text { CAV2, CAV1, COL6A6, PAK3, PDGFA, TLN2, } \\
\text { PPP1R12B, PAK5, ITGA7, RELN, PDGFD, } \\
\text { PARVA }\end{array}$ \\
\hline 04924 & Renin secretion & 6 & $<.05$ & $\begin{array}{l}\text { CLCA4, GNAI1, PDE1C, ADCYAP1R1, PDE3B, } \\
\text { NPR1 }\end{array}$ \\
\hline 04810 & $\begin{array}{l}\text { Regulation of actin } \\
\text { cytoskeleton }\end{array}$ & 10 & $\begin{array}{l}<.05 \\
0.05\end{array}$ & $\begin{array}{l}\text { FGFR1, PAK3, PDGFA, MRAS, CFL2, } \\
\text { PPP1R12B, PAK5, ITGA7, PDGFD, FGF1 }\end{array}$ \\
\hline 02010 & $\mathrm{ABC}$ transporters & 5 & $<.05$ & ABCA8, ABCA9, ABCB1, ABCA6, ABCA5 \\
\hline 04360 & Axon guidance & 7 & $\hat{0}_{0.05}$ & $\begin{array}{l}\text { SEMA5A, PAK3, SEMA3G, GNAI1, CFL2, PAK5, } \\
\text { SLIT3 }\end{array}$ \\
\hline 00350 & Tyrosine metabolism & 4 & $<.05$ & MAOA, ADH1C, ADH1B, AOC3 \\
\hline 00360 & $\begin{array}{l}\text { Phenylalanine } \\
\text { metabolism }\end{array}$ & 3 & $\begin{array}{l}<.05 \\
0.05\end{array}$ & GLYAT, MAOA, AOC3 \\
\hline 05205 & $\begin{array}{l}\text { Proteoglycans in } \\
\text { cancer }\end{array}$ & 8 & $<.05$ & $\begin{array}{l}\text { CAV2, FGFR1, CAV1, GPC3, MRAS, PPP1R12B, } \\
\text { HSPB2, FZD4 }\end{array}$ \\
\hline 04022 & $\begin{array}{l}\text { cGMP-PKG signaling } \\
\text { pathway }\end{array}$ & 7 & $<.05$ & $\begin{array}{l}\text { EDNRB, PDE2A, GNAI1, PDE3B, NPR1, CREB5, } \\
\text { ATP1A2 }\end{array}$ \\
\hline 05215 & Prostate cancer & 5 & $<.05$ & FGFR1, PDGFA, CREB5, PDGFD, TCF7L2 \\
\hline 00340 & Histidine metabolism & 3 & $\stackrel{<}{0.05}$ & ASPA, MAOA, ALDH2 \\
\hline 04964 & $\begin{array}{l}\text { Proximal tubule } \\
\text { bicarbonate } \\
\text { reclamation }\end{array}$ & 3 & $\dot{0.05}$ & CA4, ATP1A2, PCK1 \\
\hline 05032 & Morphine addiction & 5 & $\begin{array}{l}<.01 \\
0.01\end{array}$ & PDE2A, GNAI1, PDE1C, PDE3B, GRK3 \\
\hline 04922 & $\begin{array}{l}\text { Glucagon signaling } \\
\text { pathway }\end{array}$ & 5 & $<.05$ & PDE3B, CREB5, ACACB, SIK2, PCK1 \\
\hline
\end{tabular}




\begin{tabular}{|c|c|c|c|c|}
\hline 04151 & $\begin{array}{l}\text { PI3K-Akt signaling } \\
\text { pathway }\end{array}$ & 10 & $\begin{array}{l}<.05 \\
0.05\end{array}$ & $\begin{array}{l}\text { FGFR1, COL6A6, PDGFA, ITGA7, RELN, CREB5, } \\
\text { PDGFD, FGF1, GHR, PCK1 }\end{array}$ \\
\hline 04024 & $\begin{array}{l}\text { cAMP signaling } \\
\text { pathway }\end{array}$ & 7 & $<.05$ & $\begin{array}{l}\text { GNAl1, ADCYAP1R1, PDE3B, NPR1, OXTR, } \\
\text { CREB5, ATP1A2 }\end{array}$ \\
\hline 00010 & $\begin{array}{l}\text { Glycolysis / } \\
\text { Gluconeogenesis }\end{array}$ & 4 & $<.05$ & ADH1C, ALDH2, ADH1B, PCK1 \\
\hline 00982 & $\begin{array}{l}\text { Drug metabolism - } \\
\text { cytochrome P450 }\end{array}$ & 4 & $<.05$ & FMO2, MAOA, ADH1C, ADH1B \\
\hline 05218 & Melanoma & 4 & $<.05$ & FGFR1, PDGFA, PDGFD, FGF1 \\
\hline 04015 & $\begin{array}{l}\text { Rap1 signaling } \\
\text { pathway }\end{array}$ & 7 & $<.05$ & $\begin{array}{l}\text { FGFR1, GNAI1, PDGFA, TLN2, MRAS, PDGFD, } \\
\text { FGF1 }\end{array}$ \\
\hline
\end{tabular}

\section{PPI (Protein-Protein Interaction) network construction, module identification and KEGG pathway analysis}

PPI network analysis of the identified DEGs revealed that there were 334 nodes and 1127 edges interactions (Figure 3). These proteins were selected on the basis of combined score $\geq 0.4$ in STRING analysis. In addition, one significant module with a score $\geq 5$ was screened out via MCODE. The CDK1, FN1, AURKA, CCNB2, BIRC5, and TOP2A genes were identified as hub genes with highest degree of centrality in the identified module (Table 5). KEGG pathways analysis of the hub genes has been displayed in Table 6. The most significant pathways were enriched in the Oocyte meiosis, p53 signaling pathway, Progesterone-mediated oocyte maturation, and cell Cycle pathways (Table 6).

Table 5

List of identified hub genes

\begin{tabular}{|llcll|}
\hline Name of the gene & Degree of centrality & No. of nodes & Closeness of centrality \\
\hline CDK1 & 46 & 46 & 0.38288288 \\
\hline FN1 & 43 & 43 & 0.42079208 \\
\hline AURKA & 41 & & 41 & 0.4028436 \\
\hline CCNB2 & 40 & 40 & 0.38694992 \\
\hline BIRC5 & 40 & 40 & 0.38694992 \\
\hline TOP2A & 40 & 40 & 0.35027473 \\
\hline Closeness of centrality indicates how close a node is to all other nodes in the network. \\
\hline
\end{tabular}


Table 6

Kyoto encyclopedia of genes and genomes pathway analysis of hub genes related with breast cancer

$\begin{array}{lllll}\text { Pathway } & \text { Pathway Name } & \text { Gene } & \text { P- } & \text { Gene name } \\ \text { ID } & & \text { count } & \text { value } & \end{array}$

$\begin{array}{lll}04114 \text { Oocyte meiosis } 3 & <0.05 & \begin{array}{l}\text { CDK1, CCNB2, } \\ \text { AURKA }\end{array}\end{array}$

$\begin{array}{lllll}04115 & 2 & <03 \text { signaling pathway } & 2.05 & \text { CDK1, CCNB2 }\end{array}$

$04914 \quad$ Progesterone-mediated Oocyte $\quad 2 \quad<0.05 \quad$ CDK1, CCNB2

\section{Kaplan Meier Survival analysis of identified hub genes}

The prognostic value of the identified six hub genes was documented with the help of Kaplan-Meier plotter website. A high expression of CDK1 (HR 1.55 [1.25-1.92], P = 6.1e-05), FN1 (HR 0.92 [0.74-1.14], $\mathrm{P}=0.44)$, AURKA (HR 1.83 [1.47-2.28], $\mathrm{P}=3.6 \mathrm{e}-08), \mathrm{CCNB} 2$ (HR 2.06 [1.65-2.56], $\mathrm{P}=5.2 \mathrm{e}-11), \mathrm{BIRC5}$ (HR 1.52 [1.23-1.89], $P=0.00011)$, and TOP2A (HR 1.84 [1.48-2.29], $P=3.1 \mathrm{e}-08$ ]), was related to a worse OS in breast cancer patients (Figure 2). However, results of the present study also shown that high expression of FN1 (HR 0.92 [0.74-1.14], $\mathrm{P}=0.44$ ) was related with good OS in breast cancer patients but the correlation was found insignificant $(p>0.05)$ (Figure 4).

\section{Validation of hub genes participation in breast cancer: through analysis of expression profile in relation to various clinicopathological features and promoter's methylation status}

Then, the online tool UALCAN was used to document alterations, if any, in the expression levels of six hub genes in breast normal and cancerous tissues. The transcription and translation expression levels of all the hub genes were found significantly $(p<0.05)$ higher in cancerous tissues as compared to the normal tissues (Figure 5 and Supplementary data Figure 6). Further analysis of patients subgroups based on multiple clinicopathological features of breast cancer samples in the TCGA database showed consistently significant $(p<0.05)$ overexpression in the levels of the six hub genes. The expression levels of the detected hub genes were significantly $(p<0.05)$ elevated in breast cancer samples than the normal samples in patients of different cancer stages and subclasses of tumors (Supplementary data Figure 1- 
2), age, ethnicity, and menopause status of patients (Supplementary data Figures 3-5). In addition, we also explored that the expression levels of hub genes well correlated with the methylation status of the corresponding promoters. Unexpectedly, based upon up regulated expression levels, the promoter's methylation status of all the six hub genes was found to be hypermethylated (Figure 6).

\section{Hub Gene-Drug interaction network analysis}

To explore the interaction between hub genes and available therapeutic drugs of cancer, the gene-drug interaction network was constructed using CTD. As shown in Figure 9, a variety of drugs could potentially affect the expression of all the six identified hub genes including CDK1, FN1, AURKA, CCNB2, BIRC5, and TOP2A. For example, doxorubicin and bisphenol A could reduce CDK1 expression level while surmustine could elevate CDK1 expression level (Figure 7).

\section{Discussion}

Breast cancer is a group of heterogeneous diseases with multiple clinical presentations, treatment responses and subtype related to therapeutic measures. Molecular classification method of breast cancer is well recognized around the world. However, scientist gradually noticed that different molecular subtypes of breast cancer were correlated with distinct treatment responses and metastasis traits, which are useful to predict the duration of the survival and guide the proper administration of the drugs for the better treatment [16]. Till to date there is no diagnostic method available for the early detection of the breast cancer [17]. A number of therapies are available for the treatment of different breast cancer subtypes like hormonal therapy for HER-2 positive is well established. However, the response of HER2 based therapies is expected to be failure in basal-like breast cancer (BLBC) because this subtype usually expresses low or neither of these oncogenes. The BLBC expresses some normal myoepithelial cell makers such as cytokeratins (CK5/6, CK14 and CK17), HER1 and c-KIT but these markers are still unable to represent the complete characteristics of the $\operatorname{BLBC}[18,19]$.

By looking at the complete scenario, it is worth to utilize bioinformatics methods to identify the novel diagnostic and prognostic biomarkers by analyzing the online available microarray based datasets. In the present research, we retrieved the RNA microarray datasets from GEO database and identified the DEGs in breast cancer patients utilizing bioinformatics tools. We further validated the role of the identified hub genes in breast cancer development and progression by evaluating breast invasive carcinoma datasets (BRICS) of TCGA database integrated with UALCAN platform. We also aimed to construct the drug-gene interaction network and performed correlation analysis between the expression level of hub genes and the methylation status of their promoters. In total 449 DEGs were identified (151 up-regulated and 298 down-regulated genes) including 33 common up-regulated / down-regulated genes.

KEGG pathway and GO functional annotation analysis of up-regulated DEGs reveled their involvement in various pathways including 'oocyte meiosis', 'P53 signaling pathway', 'focal adhesion', 'ECM-receptor interaction', 'Rennin secretion', 'regulation of active cytoskeleton', 'ABC transporters' and 'axon guidance'. 
The role of major pathways identified through KEGG analysis like 'oocyte meiosis', 'p53 signaling pathway', 'focal adhesion' and 'ECM-receptor interaction' pathways in the development of various cancers including breast has already been reported by Sun et al. and Bao et al. [20, 21]. Similarly, the role of Rennin secretion', 'regulation of active cytoskeleton', 'ABC transporters' and 'axon guidance' pathways in breast cancer has also been suggested by Vinson et al. and Baumann et al. [22-25]. However, these individual studies were the small scale studies containing less number of samples. While the present study was performed on datasets consisting of a large sample size and higher number of target genes. Hence, it revealed a better picture of the molecular pathways contributing to the pathogenesis of breast cancer. The information obtained from this study is persistent with the existing state of knowledge, and provide further confirmation regarding the role of oocyte meiosis', 'p53 signaling pathway', 'focal adhesion', 'ECM-receptor interaction', 'Rennin secretion', 'regulation of active cytoskeleton', 'ABC transporters' and 'axon guidance' pathways in the breast cancer.

The PPI network of all the DEGs was then constructed to identify the module and hub genes. A total of 36 key genes were found in the identified module, out of which six up-regulated genes including CDK1, FN1, AURKA, CCNB2, BIRC5 and TOP2A showed the highest degree of centrality and were considered for the further analysis. KEGG pathway analysis of the identified hub genes showed their significant enrichment in terms of Oocyte meiosis, p53 signaling pathway, Progesterone-mediated oocyte maturation and Cell cycle pathways (Fig. 8).

The cell cycle dysregulation is the major event that occurs in cancer cells [26] and Cyclin-dependent kinases (CDKs) are the well-known proteins involved in the regulation of cell cycle [27]. The downregulation of CDK1 has been associated with breast cancer by Lie Ding et al. [28]. Moreover, Lie Ding et al. [28] also reported that breast cancer patients with overexpression of CDK1 and CDK2 had significantly poorer OS compared to those with low CDK1 and CDK2 expression. Similar role of CDKs has also been reported in renal cell carcinoma [29]. Izadi et al. [30] revealed that down-regulation of CDK1 leads to the increased cell proliferation in breast cancer. They further added that the selective blockage of CDK1 alone or in combination with other therapeutics leads to potent anti-cancer outcomes. Contrary to the earlier reports we investigated the overexpression of CDK1 in breast cancer which suggests that elevated expression of CDK1 may serve as novel potential biomarkers for predicting the outcome of breast cancer.

FN1 is an extracellular matrix glycoprotein which is involved in cell proliferation, embryogenesis, wound healing, host defense, epithelial-mesenchymal transition (EMT), metastasis, and oncogenic transformation [31]. Previous reports revealed its involvement in the pathogenesis of various tumors and up-regulation in multiple cancer types, such as esophageal squamous cell carcinoma, colorectal cancer (CLC), and thyroid cancer [32-34]. Elevated expression and accumulation of FN1 has been reported in vincristine-resistant myeloma cells, while in other ECM components, including type II collagen a1, its level was found to be down-regulated [35]. However, the role of FN1 is not extensively studied in breast cancer. Until now, only a single study [36] has identified the role of FN1 overexpression in breast cancer and suggested it as a potential biomarker in breast cancer. Our findings are similar to this report and further validate the conclusions of Wang et al. [36]. 
AURKA is an important molecule for cell cycle progression. It has been frequently found overexpressed or mutated in various types of human tumor $[37,38]$. However, its role in breast cancer is still uncertain due to the conflicting results of the various studies For example, Tanaka et al. [39] investigated 33 cases of breast cancer and found overexpression of AURKA in $94 \%$ of the cases. Miyoshi et al. observed AURKA overexpression in $64 \%$ of the breast cancer cases using reverse transcription polymerase chain reaction (RT-PCR) in a group of 47 patients [40]. However, comparatively a larger study including 112 breast cancer patients did not find any association between AURKA expression and patient's survival [41]. However, Nadler et al. observed elevated expression of AURKA in breast tumors and related it with OS [42]. In our study we also observed the overexpression of AURKA and thus suggest it as a potential biomarker of breast cancer.

Cyclin B2 (CCNB2) is a member of cyclin family. The major function of CCNB2 is to regulate the cell cycle by promoting the activation of CDC2 kinase in eukaryotes, and inhibition of CCNB2 induced cell cycle arrest. Various studies had reported its overexpression in multiple types of cancers including bladder cancer, uterine corpus endometrial carcinoma, prostate cancer, and gastric cancer [43, 44]. In breast cancer, CCNB2 protein levels have been reported as an independent prognostic factor which significantly correlated with a poor disease specific survival [45]. Our results indicated up-regulation of CCNB2 in breast cancer samples as compared to the normal controls and highlighted it as potential biomarker in breast cancer. However, the underlying mechanisms of CCNB2 on tumor progression need to be further investigated.

BIRC5 (Survivin) is a multifunctional protein that controls rate of mitosis and participates in the regulation of apoptosis, and the cellular stress response [46]. Overexpression of BIRC5 in breast cancer is associated with increased resistance to chemotherapy, radiotherapy, increased grade and tumour size, as well as with the decreased survival rate [47]. According to Marsicano et a/ breast cancer patients with higher expression of BIRC5 have significantly greater chance of developing metastasis than patients with lower expression [48]. Similar results were observed during present study which suggested that BIRC5 overexpression could be a potential prognostic and diagnostic biomarker in breast cancer.

DNA topoisomerase 2-alpha (TOP2A) gene encodes a protein that controls and alters topologic states of DNA during the process of transcription. TOP2A is a significant prognostic factor in predicting the breast cancer patients' OS, and low expression of TOP2A has been associated with a better clinical outcome [49]. In addition TOP2A amplification or deletion is also known to enhance responsiveness to anthracycline-containing chemotherapy regimens relative to non-anthracycline regimens [50]. Various other studies have evaluated the predictive value of TOP2A using different methodologies and inconsistent results related with TOP2A differential expression analysis have been reported. Indeed, the correlation among techniques employed for the assessment of TOP2A status has not been well evaluated [51]. However, in the light of our results we conclude that the elevated expression of TOP2A could be a prognostic factor for breast cancer and indicates a role of anthracycline-containing chemotherapy regimens for effective treatment of the disease. 
The survival data obtained from Kaplan-Meier method showed that the OS of breast cancer patients harboring high expression of CDK1, AURKA, CCNB2, BIRC5 and TOP2A genes was significantly worsened than that of low-expression patients. However, the elevated expression of FN1 was found insignificantly $(p<0.05)$ correlated with good OS of breast cancer patients which need to be further validated through voluminous testing.

The elevated expression levels of identified hub genes were further validated on all the BRIC datasets present of TCGA database through UALCAN platform. Results revealed the persistent significant $(p<0.05)$ up-regulation of transcription and translation level of the all the hub genes in breast cancer samples including various subgroups of patients categorized on the basis of various clinicopathological features (i.e., tumor stage, breast cancer major subclasses, age, race and menopause status) as compared to the normal controls.

A correlation between expression levels of all the hub genes and the methylation status of their promoters was also accessed and it was found that the expression levels of hub genes have a significant $(p<0.05)$ positive correlation with the methylation extent of their corresponding promoters. Here, the methylation scenario challenges the classical view of the methylation where the up-regulation was always negatively correlated with the expression level. However, further studies need to validate these findings.

We further studied the response of available therapeutics drugs in regulating the expression levels of identified six hub genes. The information thus generated can serve as a guide during the treatment of patients exhibiting up-regulated expression of these hub genes and selection of appropriate drugs. Whether breast cancer patients with over-expression of these hub genes will get any benefit from the suppression of hub genes, or whether these hub genes are promising therapeutic targets still need additional experimental support through voluminous pre-clinical and clinical trials.

Currently, few relevant studies based on the same datasets that we used in the present study have been published. In one such study, Jin et al. [52] analyzed the microarray dataset GSE10810 in a consortium with few other datasets and identified 15 different hub genes by constructing PPI network. They also analyzed the effect of hub genes expression on the survival duration of patients. Similarly the dataset GSE29431 was also analyzed by Lin et al. [53] along with two different other datasets and they identified 10 hub genes in that study. Compared to their results, the hub genes we identified in the present study are not exactly compatible with already identified hub genes. The unique combinations of these datasets and different analysis methods have been used in the current study, which might partly account for the reasons for these differences. Some advantages of our study mainly lie in the following points: First of all we validated the expression of all the identified hub genes on BRIC datasets present on TCGA database and further investigated the status of hub genes expression in different sub groups of patients based upon various clinicopathological features such as breast cancer stages, major subclasses, age, race, and menopause status. Secondly, we performed the correlational analysis between the expression of hub genes and their respective promoters' methylation statuses. Finally, exploring the impact of many 
commonly used chemotherapeutic drugs on regulation of the hub genes expression may potentially contribute to identify new biomarkers and targets for better management of breast cancer.

\section{Conclusion}

The present study provided a comprehensive overview of DEGs and associated biological processes which are dysregulated and may be involved in breast cancer progression. We documented up-regulation of the six hub genes including CDK1, FN1, AURKA, CCNB2, BIRC5, and TOP2A and propose that the detected hub genes are the most likely players which can be linked with breast cancer development and progression. The validation of hub genes overexpression in TCGA BRIC patients having diverse clinicopathological parameters further reinforced the role of these hub genes in pathogenesis of breast cancer. Results also revealed the higher expression levels of hub genes as a poor clinical outcome in breast cancer patients. The present study has significantly enhanced our understanding regarding breast cancer pathogenesis and progression. Moreover, the findings of the present study have provided new insights into the diagnosis, prognosis and treatment of breast cancer. However, the specific functions of the identified genes in breast cancer should be confirmed by further molecular biological investigations.

\section{List Of Abbreviations}

$\mathrm{BC}=$ Breast cancer

$\mathrm{ER}=$ Estrogen receptor

$\mathrm{PR}=$ Progesterone receptor

EGF = Epidermal growth factor

HER2 = Human epidermal growth factor receptor 2

$\mathrm{GEO}=$ Gene Omnibus

CTD $=$ Comparative Toxicogenomics Database

$\mathrm{GO}=$ Gene ontology

KEGG = Kyoto Encyclopedia of Genes and Genomes

GEGs = differentially expressed genes

PPI = Protein-Protein Interaction

$\mathrm{KM}=$ Kaplan-Meier

CDKS $=$ Cyclin-dependent kinases 
CCNB2 $=$ Cyclin B2

\section{Declarations}

\section{Ethics approval and consent to participate}

NA

\section{Consent for publication}

NA

\section{Availability of data and materials}

The two datasets (GSE10810 and GSE61304) on which the conclusion of present study based are available in GEO database (at: https://www.ncbi.nlm.nih.gov/geo/)

\section{Competing interests}

None

\section{Funding}

The work presented in this manuscript was supported by the NRPU Project No.

9481/Punjab/NRPU/R\&D/HEC/2017 funded by Higher Education Commission, Pakistan.

\section{Authors' contributions}

All authors contributed to the study conception and design. Material preparation, data collection and analysis were performed by [Yasir Hameed], and [Samina Ejaz]. The first draft of the manuscript was written by [Yasir Hameed] which was finalized by [Samina Ejaz], all the authors commented on previous versions of the manuscript. All authors read and approved the final manuscript.

\section{Acknowledgements}

NA

References 
1. Siegel RL, Miller KD, Jemal A. Cancer statistics, 2019. CA Cancer J Clin 2019:691:7-34.

2. Kast K, Link T, Friedrich K, Petzold A, Niedostatek A, Schoffer O, Werner C, Klug SJ, Werner A, Gatzweiler A, et al: Impact of breast cancer subtypes and patterns of metastasis on outcome. Breast Cancer Res Treat 2015:1503:621-629.

3. Coates AS, Winer EP, Goldhirsch A, Gelber RD, Gnant M, Piccart-Gebhart M, Thürlimann B, Senn H-J, Members $\mathrm{P}$, André F. Tailoring therapies-improving the management of early breast cancer: St Gallen International Expert Consensus on the Primary Therapy of Early Breast Cancer 2015. Annals of oncology 2015:268:1533-1546.

4. Mohammed ZM, McMillan DC, Edwards J, Mallon E, Doughty JC, Orange C, Going JJ. The relationship between lymphovascular invasion and angiogenesis, hormone receptors, cell proliferation and survival in patients with primary operable invasive ductal breast cancer. $B M C$ clinical pathology 2013:131:31.

5. Zhang J, Bing Z, Yan P, Tian J, Shi X, Wang Y, Yang K. Identification of 17 mRNAs and a miRNA as an integrated prognostic signature for lung squamous cell carcinoma. The Journal of Gene Medicine 2019:218:e3105.

6. Liu B-X, Huang G-J, Cheng H-B. Comprehensive analysis of core genes and potential mechanisms in rectal cancer. Journal of Computational Biology 2019:2611:1262-1277.

7. Mok SC, Bonome T, Vathipadiekal V, Bell A, Johnson ME, Park D-C, Hao K, Yip DK, Donninger H, Ozbun L. A gene signature predictive for outcome in advanced ovarian cancer identifies a survival factor: microfibril-associated glycoprotein 2. Cancer cell 2009:166:521-532.

8. Bowen NJ, Walker LD, Matyunina LV, Logani S, Totten KA, Benigno BB, McDonald JF. Gene expression profiling supports the hypothesis that human ovarian surface epithelia are multipotent and capable of serving as ovarian cancer initiating cells. BMC Med Genom. 2009;21:71.

9. Elgaaen BV, Olstad OK, Haug KBF, Brusletto B, Sandvik L, Staff AC, Gautvik KM, Davidson B. Global miRNA expression analysis of serous and clear cell ovarian carcinomas identifies differentially expressed miRNAs including miR-200c-3p as a prognostic marker. BMC cancer 2014:141:80.

10. Dennis G Jr, Sherman BT, Hosack DA, Yang J, Gao W, Lane HC, Lempicki RA. DAVID: Database for Annotation, Visualization, and Integrated Discovery. Genome Biology 2003:49:R60-R60.

11. Szklarczyk D, Franceschini A, Wyder S, Forslund K, Heller D, Huerta-Cepas J, Simonovic M, Roth A, Santos A, Tsafou KP. STRING v10: protein-protein interaction networks, integrated over the tree of life. Nucleic acids research 2015:43D1:D447-D452.

12. Szász AM, Lánczky A, Nagy Á, Förster S, Hark K, Green JE, Boussioutas A, Busuttil R, Szabó A, Győrffy B. Cross-validation of survival associated biomarkers in gastric cancer using transcriptomic data of 1,065 patients. Oncotarget. 2016;731:49322.

13. Lacny S, Wilson T, Clement F, Roberts DJ, Faris P, Ghali WA, Marshall DA. Kaplan-Meier survival analysis overestimates cumulative incidence of health-related events in competing risk settings: a meta-analysis. Journal of Clinical Epidemiology 2018:93:25-35. 
14. Chandrashekar DS, Bashel B, Balasubramanya SAH, Creighton CJ, Ponce-Rodriguez I, Chakravarthi BVSK, Varambally S. UALCAN: A Portal for Facilitating Tumor Subgroup Gene Expression and Survival Analyses. Neoplasia 2017:198:649-658.

15. Mattingly CJ, Colby GT, Forrest JN, Boyer JL. The Comparative Toxicogenomics Database (CTD). Environmental health perspectives 2003:1116:793-795.

16. Lehmann BD, Bauer JA, Chen X, Sanders ME, Chakravarthy AB, Shyr Y, Pietenpol JA. Identification of human triple-negative breast cancer subtypes and preclinical models for selection of targeted therapies. The Journal of clinical investigation 2011:1217:2750-2767.

17. Unger-Saldaña K. Challenges to the early diagnosis and treatment of breast cancer in developing countries. World journal of clinical oncology 2014:53:465-477.

18. Nielsen TO, Hsu FD, Jensen K, Cheang M, Karaca G, Hu Z, Hernandez-Boussard T, Livasy C, Cowan D, Dressler L. Immunohistochemical and clinical characterization of the basal-like subtype of invasive breast carcinoma. Clinical cancer research 2004:1016:5367-5374.

19. Carey LA, Perou CM, Livasy CA, Dressler LG, Cowan D, Conway K, Karaca G, Troester MA, Tse CK, Edmiston S. Race, breast cancer subtypes, and survival in the Carolina Breast Cancer Study. Jama 2006:29521:2492-2502.

20. Sun Y, Yuan K, Zhang P, Ma R, Zhang QW, Tian XS. Crosstalk analysis of pathways in breast cancer using a network model based on overlapping differentially expressed genes. Experimental and therapeutic medicine 2015:102:743-748.

21. Bao Y, Wang L, Shi L, Yun F, Liu X, Chen Y, Chen C, Ren Y, Jia Y. Transcriptome profiling revealed multiple genes and ECM-receptor interaction pathways that may be associated with breast cancer. Cellular \& molecular biology letters 2019:241:38.

22. Vinson GP, Barker S, Puddefoot JR. The renin-angiotensin system in the breast and breast cancer. Endocr Relat Cancer 2012:191:11-0335.

23. Chen S, Hourwitz MJ, Campanello L, Fourkas JT, Losert W, Parent CA. Actin Cytoskeleton and Focal Adhesions Regulate the Biased Migration of Breast Cancer Cells on Nanoscale Asymmetric Sawteeth. ACS Nano 2019:132:1454-1468.

24. Balaji SA, Udupa N, Chamallamudi MR, Gupta V, Rangarajan A. Role of the Drug Transporter ABCC3 in Breast Cancer Chemoresistance. PloS one 2016:115:e0155013-e0155013.

25. Baumann J, Sevinsky C, Conklin DS. Lipid biology of breast cancer. Biochimica et biophysica acta 2013:183110:1509-1517.

26. Evan GI, Vousden KH. Proliferation, cell cycle and apoptosis in cancer. Nature. 2001;4116835:342-8.

27. Malumbres M, Barbacid M. Cell cycle, CDKs and cancer: a changing paradigm. Nat Rev Cancer 2009:93:153-166.

28. Ding L, Cao J, Lin W, Chen H, Xiong X, Ao H, Yu M, Lin J, Cui Q. The Roles of Cyclin-Dependent Kinases in Cell-Cycle Progression and Therapeutic Strategies in Human Breast Cancer. Int J Mol Sci 2020:216. 
29. Hongo F, Takaha N, Oishi M, Ueda T, Nakamura T, Naitoh Y, Naya Y, Kamoi K, Okihara K, Matsushima T, et al: CDK1 and CDK2 activity is a strong predictor of renal cell carcinoma recurrence. Urol Oncol 2014:328:1240-1246.

30. Izadi S, Nikkhoo A, Hojjat-Farsangi M, Namdar A, Azizi G, Mohammadi H, Yousefi M, Jadidi-Niaragh F. CDK1 in Breast Cancer: Implications for Theranostic Potential. Anticancer Agents Med Chem 2020:310:1871520620666200203125712.

31. Pankov R, Yamada KM. Fibronectin at a glance. J Cell Sci 2002:115Pt 20:3861-3863.

32. Xiao J, Yang W, Xu B, Zhu H, Zou J, Su C, Rong J, Wang T, Chen Z. Expression of fibronectin in esophageal squamous cell carcinoma and its role in migration. BMC Cancer 2018:181:018-4850.

33. Nakagawa Y, Nakayama H, Nagata M, Yoshida R, Kawahara K, Hirosue A, Tanaka T, Yuno A, Matsuoka Y, Kojima T, et al: Overexpression of fibronectin confers cell adhesion-mediated drug resistance (CAM-DR) against 5-FU in oral squamous cell carcinoma cells. Int J Oncol 2014:444:1376-1384.

34. Sponziello M, Rosignolo F, Celano M, Maggisano V, Pecce V, De Rose RF, Lombardo GE, Durante C, Filetti S, Damante G, et al: Fibronectin-1 expression is increased in aggressive thyroid cancer and favors the migration and invasion of cancer cells. Mol Cell Endocrinol 2016:431:123-132.

35. Mutlu P, Ural AU, Gündüz U. Differential gene expression analysis related to extracellular matrix components in drug-resistant RPMI-8226 cell line. Biomed Pharmacother 2012:663:228-231.

36. Wang Y, Xu H, Zhu B, Qiu Z, Lin Z. Systematic identification of the key candidate genes in breast cancer stroma. Cellular \& Molecular Biology Letters 2018:231:44.

37. Keen N, Taylor S. Aurora-kinase inhibitors as anticancer agents. Nature Reviews Cancer 2004:412:927-936.

38. Fu J, Bian M, Jiang Q, Zhang C. Roles of Aurora kinases in mitosis and tumorigenesis. Mol Cancer Res. 2007;51:1-10.

39. Tanaka T, Kimura M, Matsunaga K, Fukada D, Mori H, Okano Y: Tanaka T, Kimura M, Matsunaga K, Fukada D, Mori H, Okano Y. Centrosomal kinase AIK1 is overexpressed in invasive ductal carcinoma of the breast. Cancer Res 59: 2041-2044. Cancer research 1999:59:2041-2044.

40. Miyoshi Y, Iwao K, Egawa C, Noguchi S. Association of centrosomal kinase STK15/BTAK mRNA expression with chromosomal instability in human breast cancers. Int J Cancer 2001:923:370-373.

41. Royce ME, Xia W, Sahin AA, Katayama H, Johnston DA, Hortobagyi G, Sen S, Hung MC. STK15/Aurora-A expression in primary breast tumors is correlated with nuclear grade but not with prognosis. Cancer 2004:1001:12-19.

42. Nadler Y, Camp RL, Schwartz C, Rimm DL, Kluger HM, Kluger Y. Expression of Aurora A (but not Aurora B) is predictive of survival in breast cancer. Clin Cancer Res. 2008;1414:4455-62.

43. Huang CG, Li FX, Pan S, Xu CB, Dai JQ, Zhao XH. Identification of genes associated with castrationresistant prostate cancer by gene expression profile analysis. Mol Med Rep 2017:165:6803-6813. 
44. Shi Q, Wang W, Jia Z, Chen P, Ma K, Zhou C. ISL1, a novel regulator of CCNB1, CCNB2 and C-MYC genes, promotes gastric cancer cell proliferation and tumor growth. Oncotarget 2016:724:3648936500.

45. Shubbar E, Kovács A, Hajizadeh S, Parris TZ, Nemes S, Gunnarsdóttir K, Einbeigi Z, Karlsson P, Helou $\mathrm{K}$. Elevated cyclin B2 expression in invasive breast carcinoma is associated with unfavorable clinical outcome. BMC Cancer 2013:131:1471-2407.

46. Groner B, Weiss A. Targeting survivin in cancer: novel drug development approaches. BioDrugs 2014:281:27-39.

47. Altieri DC. Validating survivin as a cancer therapeutic target. Nat Rev Cancer 2003:31:46-54.

48. Marsicano SR, Kuniyoshi RK, Gehrke FS, Alves BC, Azzalis LA, Fonseca FL. Survinin expression in patients with breast cancer during chemotherapy. Tumour Biol. 2015;365:3441-5.

49. Xu YC, Zhang FC, Li JJ, Dai JQ, Liu Q, Tang L, Ma Y, Xu Q, Lin XL, Fan HB, et al: RRM1, TUBB3, TOP2A, CYP19A1, CYP2D6: Difference between mRNA and protein expression in predicting prognosis of breast cancer patients. Oncol Rep 2015:344:1883-1894.

50. O'Malley FP, Chia S, Tu D, Shepherd LE, Levine MN, Bramwell VH, Andrulis IL, Pritchard KI.

Topoisomerase II alpha and responsiveness of breast cancer to adjuvant chemotherapy. Journal of the National Cancer Institute 2009:1019:644-650.

51. Romero A, Martín M, Cheang MC, López García-Asenjo JA, Oliva B, He X, de la Hoya M, García Sáenz J, Arroyo Fernández M, Díaz Rubio E, et al: Assessment of Topoisomerase Il a status in breast cancer by quantitative PCR, gene expression microarrays, immunohistochemistry, and fluorescence in situ hybridization. Am J Pathol 2011:1784:1453-1460.

52. Jin H, Huang X, Shao K, Li G, Wang J, Yang H, Hou Y. Integrated bioinformatics analysis to identify 15 hub genes in breast cancer. Oncology letters 2019:182:1023-1034.

53. Lin Y, Fu F, Lv J, Wang M, Li Y, Zhang J, Wang C. Identification of potential key genes for HER-2 positive breast cancer based on bioinformatics analysis. Medicine. 2020;991:e18445-5.

54. Chow JPH, Poon RYC, Ma HT. Inhibitory Phosphorylation of Cyclin-Dependent Kinase 1 as a Compensatory Mechanism for Mitosis Exit. Molecular and Cellular Biology 2011:317:1478-1491.

55. Li D, Hu C, Li H. Survivin as a novel target protein for reducing the proliferation of cancer cells (Review). Biomed Rep 2018:85:399-406.

56. Willems E, Dedobbeleer M, Digregorio M, Lombard A, Lumapat PN, Rogister B. The functional diversity of Aurora kinases: a comprehensive review. Cell Div 2018:13:7-7.

57. Gao S-Y, Li E-M, Cui L, Lu X-F, Meng L-Y, Yuan H-M, Xie J-J, Du Z-P, Pang J-X, Xu L-Y. Sp1 and AP-1 regulate expression of the human gene VIL2 in esophageal carcinoma cells. $J$ Biol Chem 2009:28412:7995-8004.

\section{Figures}


$\underline{\text { Biological process }}$

Gene count

Cell division Nuclear division

Organelle fission

Mitotic cell cycle process

Mitotic nuclear division

Cell cycle process

Chromosome segregation

Sister chromatid segregation

Cellular component organization

Chromosome organization

Biogensis

Regulation of cell cycle

Regulation of mitotic cell cycle

Cell cycle checkpoint

Single-organism organelle transition

Mitotic cell cycle phase transition

Mitotic sister chromatid segregation

Organelle organization

Microtubule cytoskeleton organization

DNA metabolic process

(A) $\underline{\text { Biological process }}$

$\underline{\text { Gene count }}$

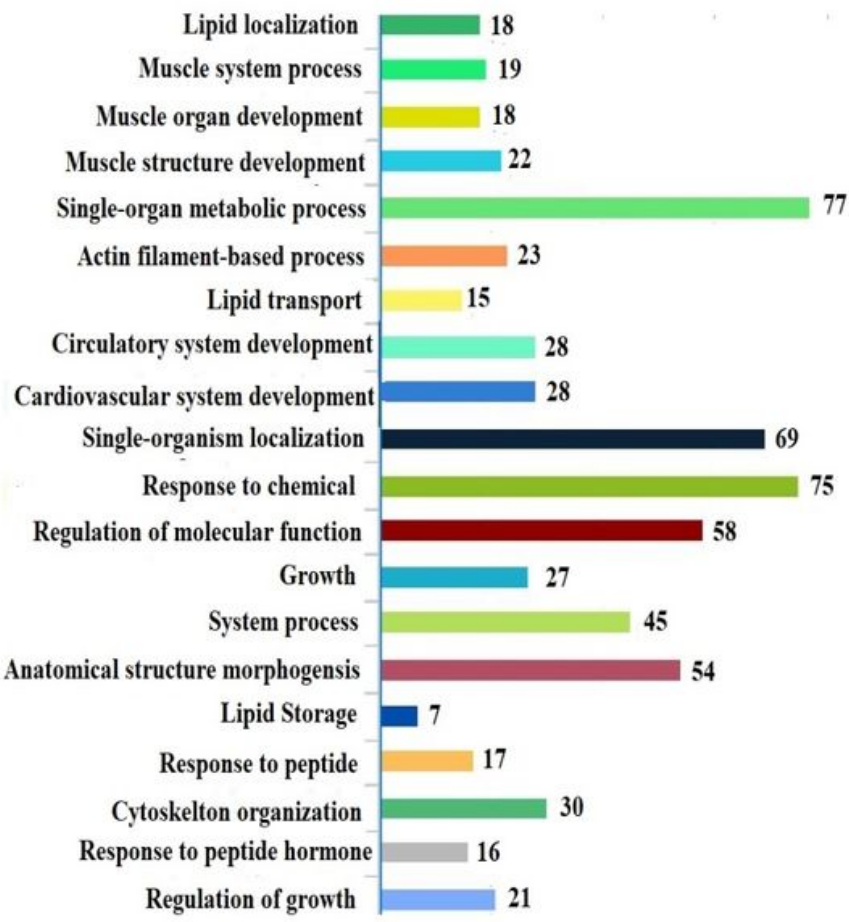

(B)

\section{Figure 1}

Enriched GO functions analysis results of DEGs. (A) Up-regulated genes GO analysis. (B) Down-regulated genes $\mathrm{GO}$ analysis. $\mathrm{GO}$ = gene ontology; DEGs = differentially expressed genes.

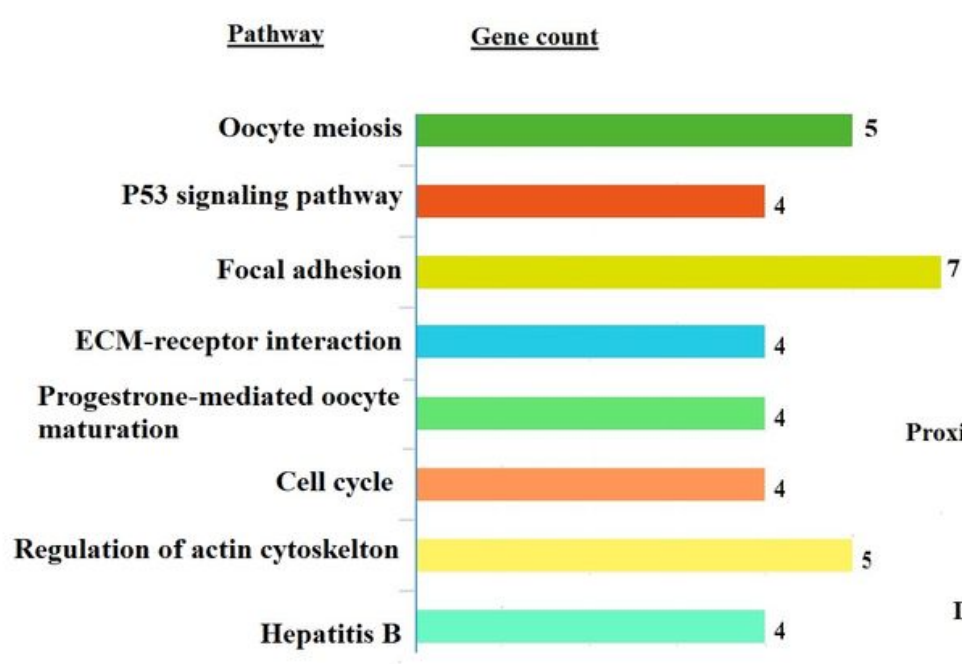

(A)

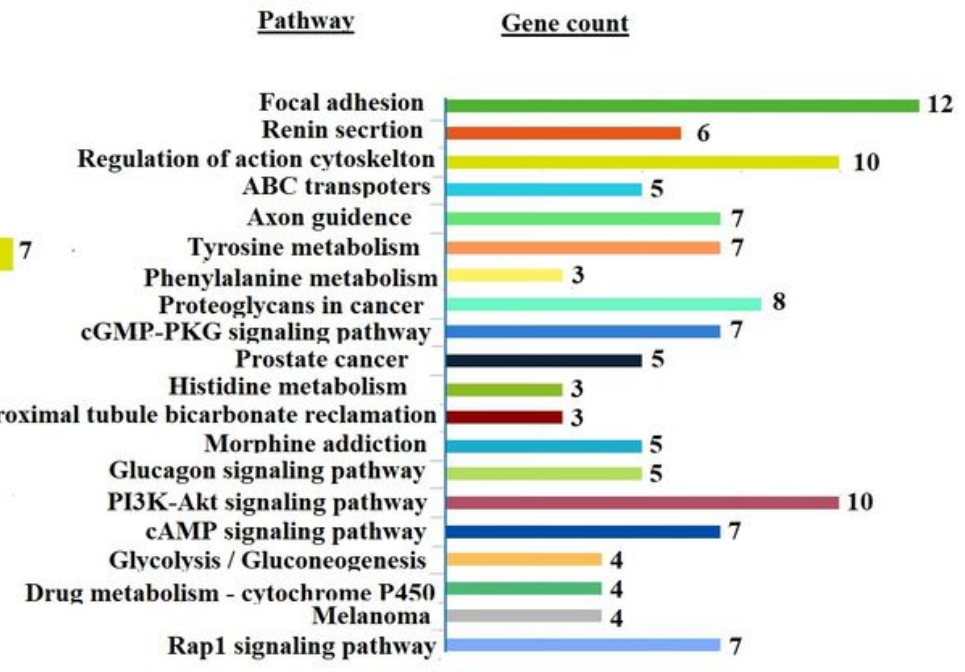

(B)

Figure 2 
KEGG pathway analysis results of differentially expressed genes. (A) Up-regulated genes KEGG pathway analysis results. (B) Down-regulated genes KEGG pathway analysis results. KEGG = Kyoto Encyclopedia of Genes and Genomes.

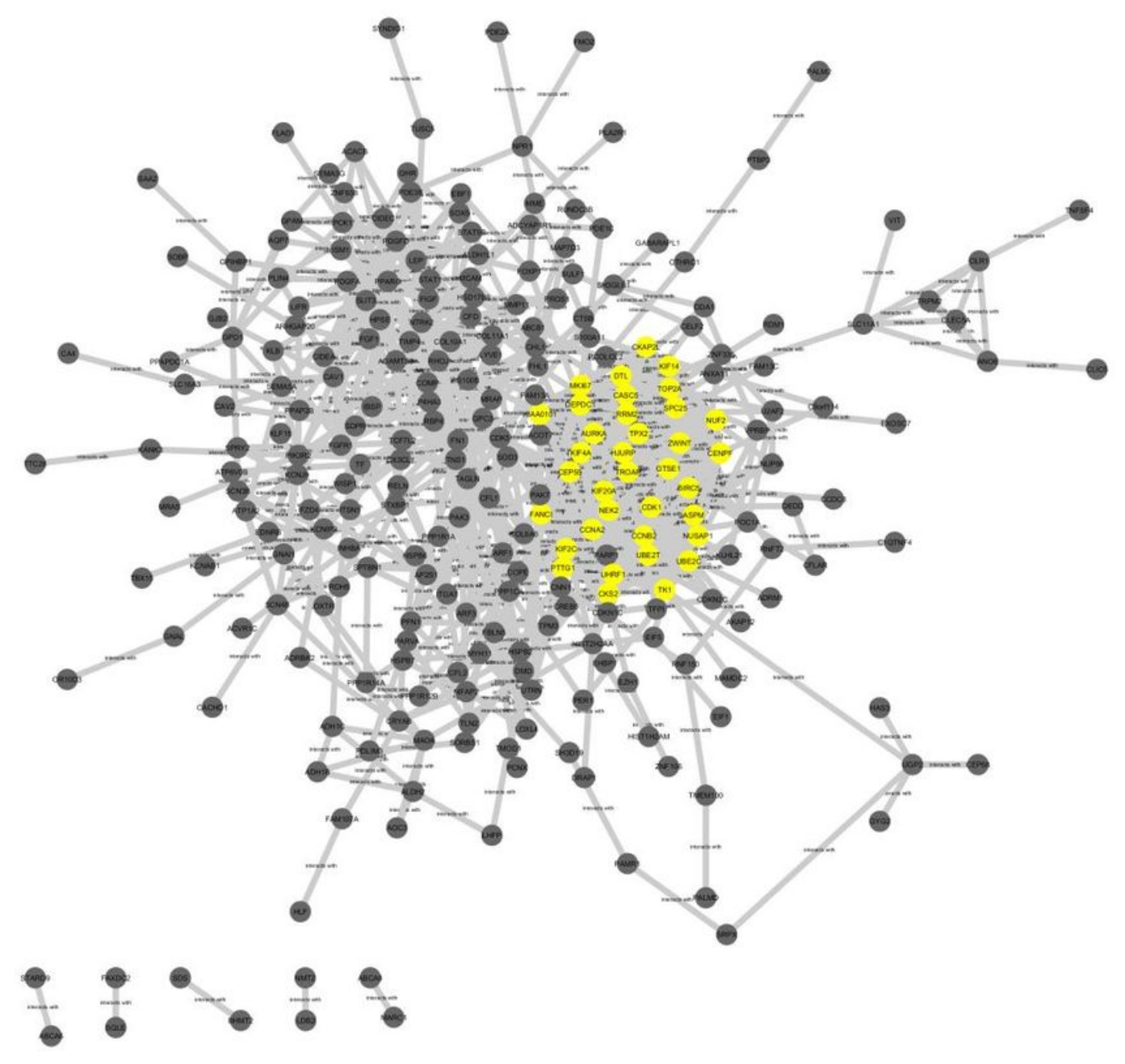

Figure 3

protein-protein interaction network of all the DEGs Yellow color indicates the main identified module containing CDK1, FN1, AURKA, CCNB2, BIRC5, and TOP2A hub genes. $p$-value $(p<0.05)$ was used to represent the significance of the correlation between the observed parameters. 
A

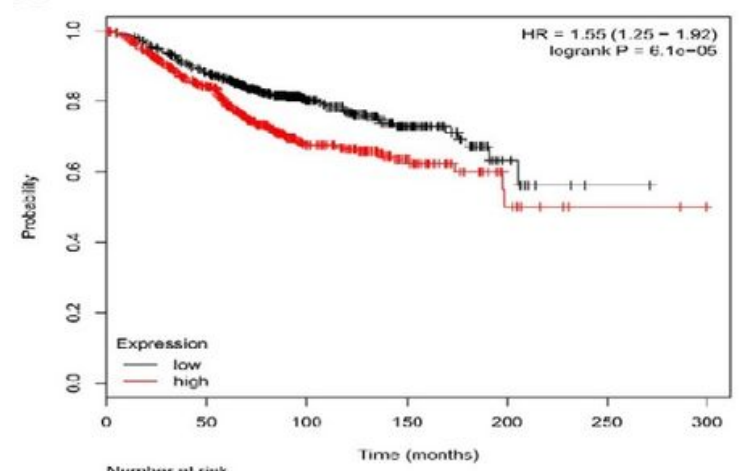

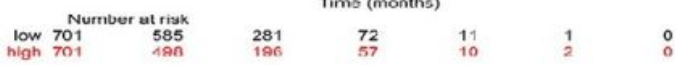

D

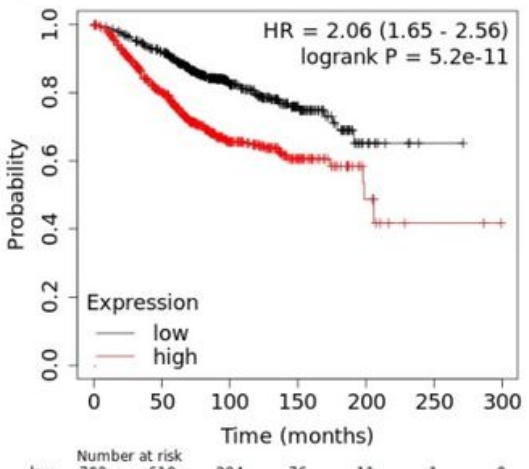

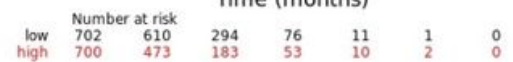

B

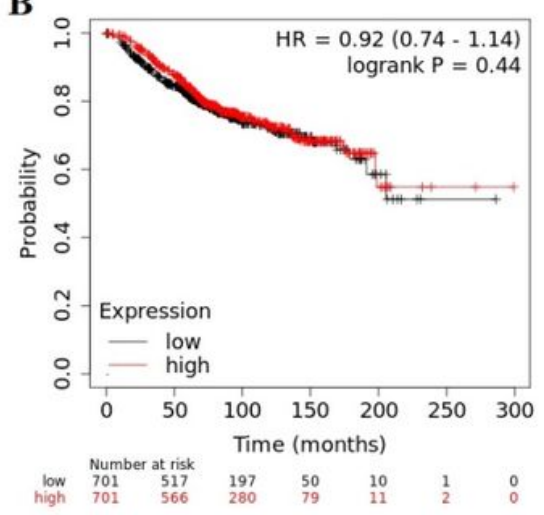

E

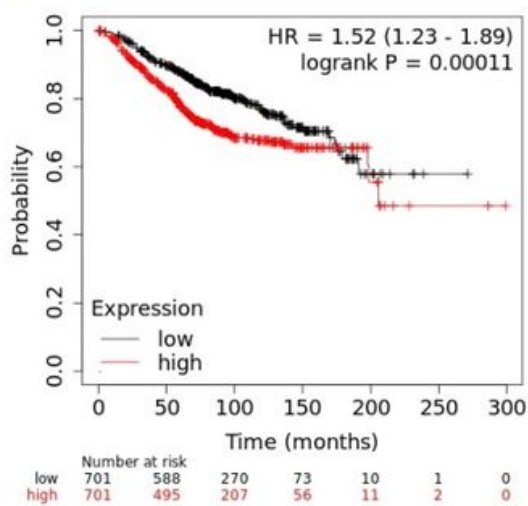

C

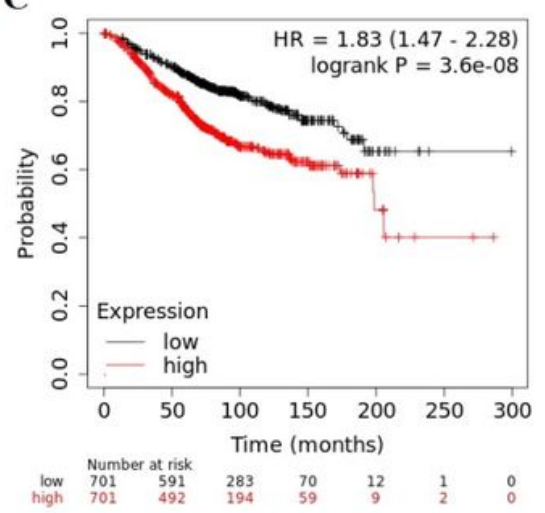

F

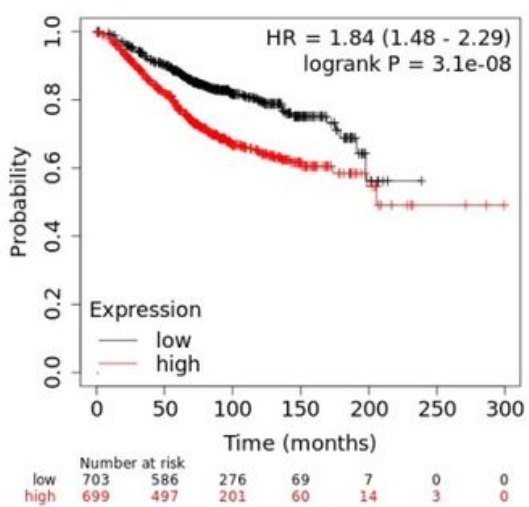

\section{Figure 4}

Kaplan-Meier survival curves of six hub genes in breast cancer patients. Overall survival (OS) by low and high level of (A) CDK1, (B) FN1, (C) AURKA, (D) CCNB2, (E) BIRC5, and (F) TOP2A expression. p-value $(p<0.05)$ was used to represent the significance of the correlation between the observed parameters.
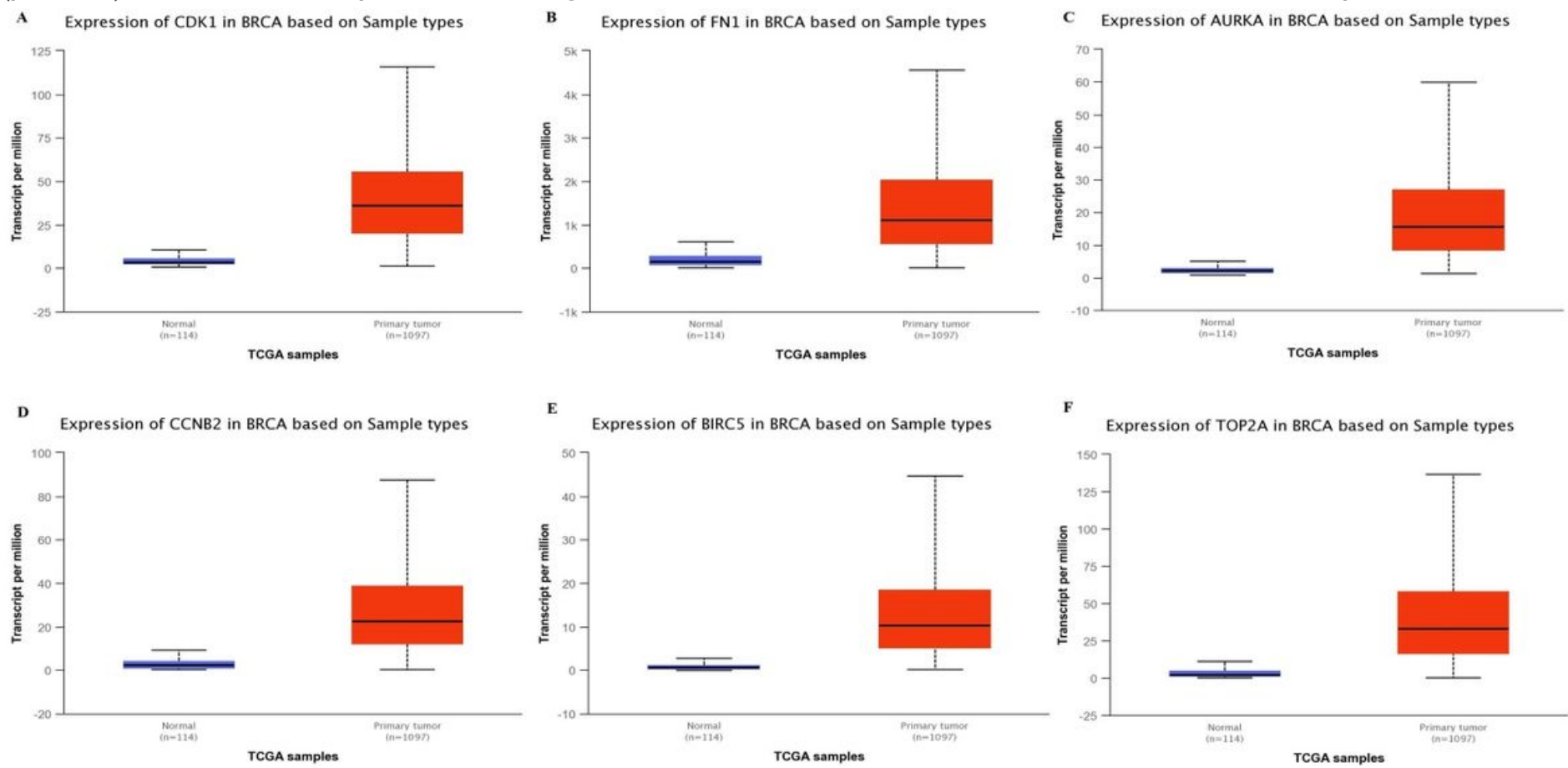


\section{Figure 5}

Relative expression of six hub genes in normal tissues and breast cancer tissues. (A) CDK1, (B) FN1, (C) AURKA, (D) CCNB2, (E) BIRC5, and (F) TOP2A. $p$-value $(p<0.05)$ was used to represent the significance of the correlation between the observed parameters.
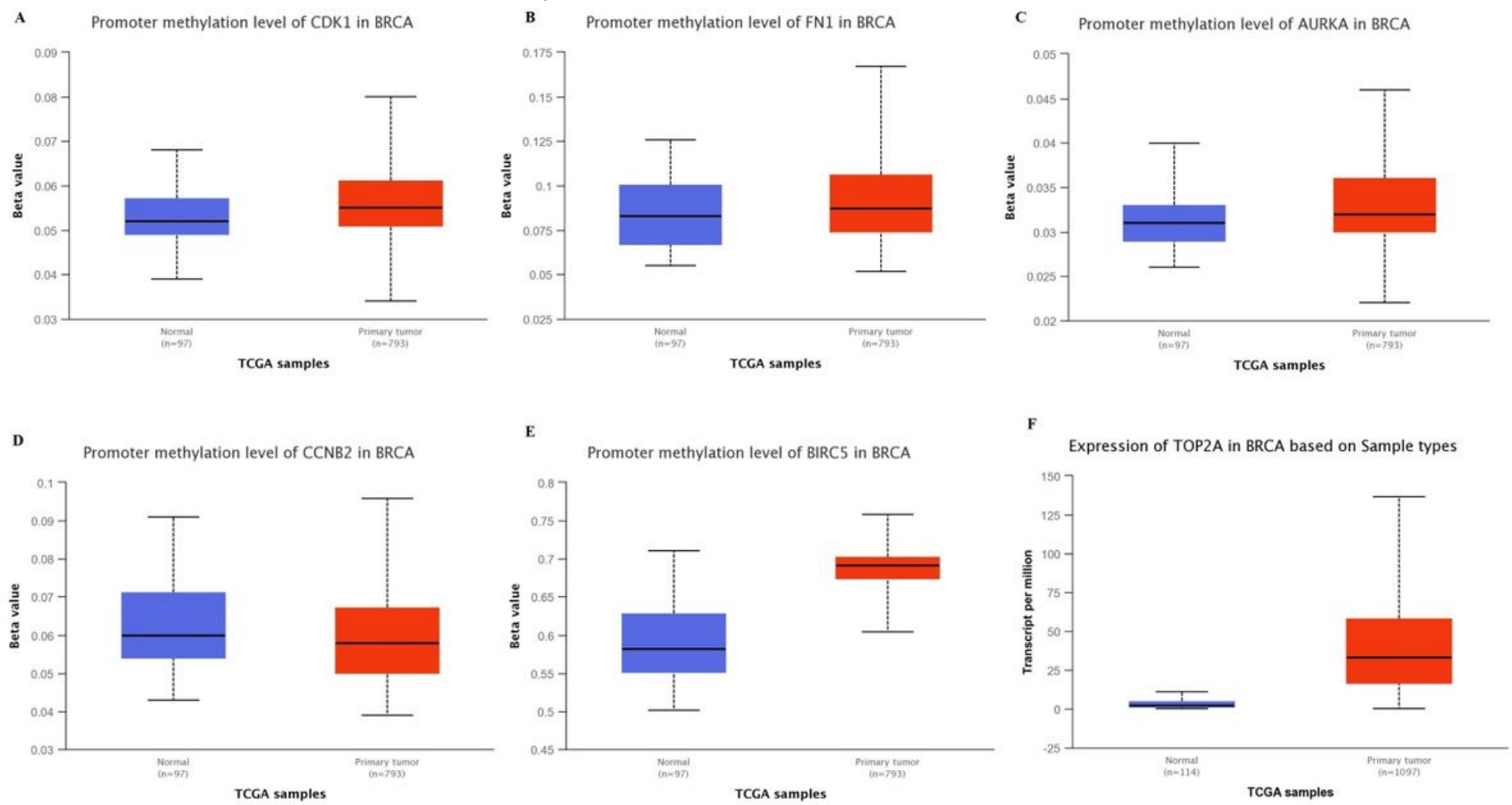

Figure 6

Relative methylation status of six hub genes in normal individual and breast cancer patients. (A) CDK1, (B) FN1, (C) AURKA, (D) CCNB2, (E) BIRC5, and (F) TOP2A. p-value $(p<0.05)$ was used to represent the significance of the correlation between the observed parameters. 


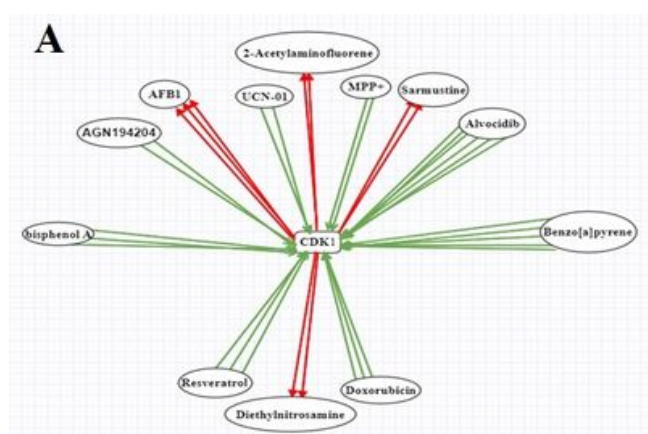

D

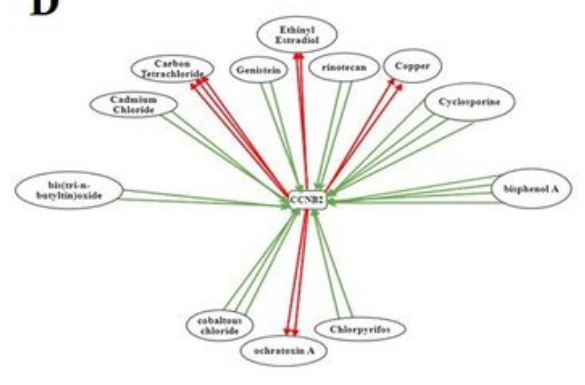

B

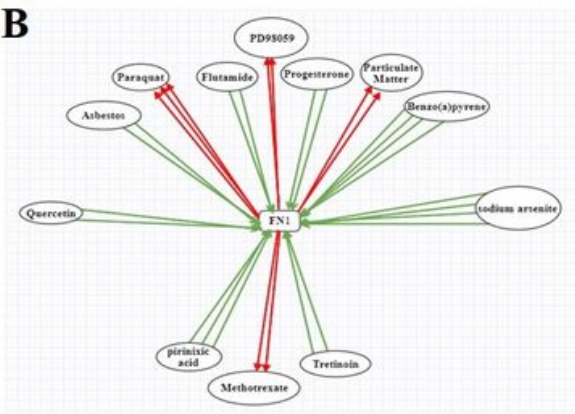

$\mathbf{E}$

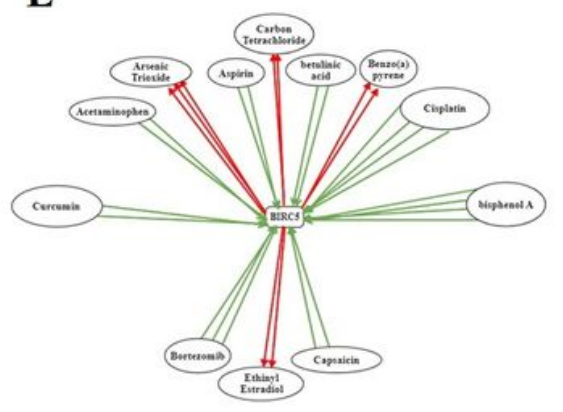

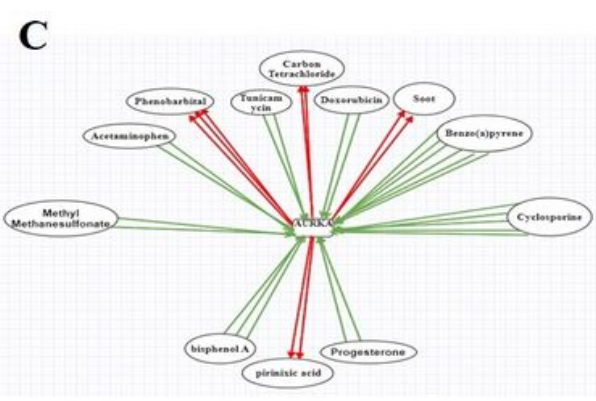

$\mathbf{F}$

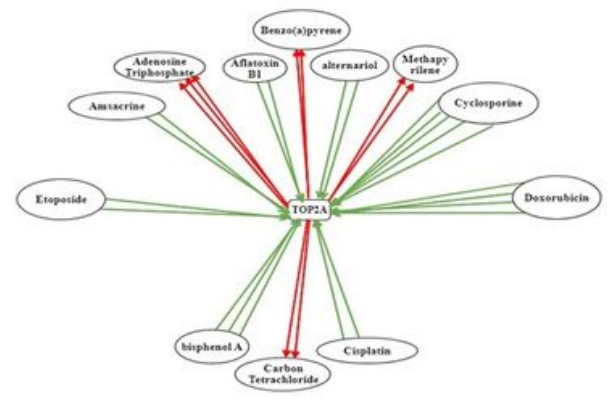

\section{Figure 7}

Gene-drug interaction network constructed with six hub genes and chemotherapeutic drugs. Panels A-F indicates available chemotherapeutic drugs that decrease or increase the expression levels of hub genes at mRNA or protein level. (A) CDK1, (B) FN1, (C) AURKA, (D) CCNB2, (E) BIRC5, and (F) TOP2A. Red arrows: chemotherapeutic drugs increase the expression of hub genes; green arrows: chemotherapeutic drugs decrease the expression of hub genes. The numbers of arrows between chemotherapeutic drugs and hub genes in the network represent the supported numbers of literatures by previous studies. 


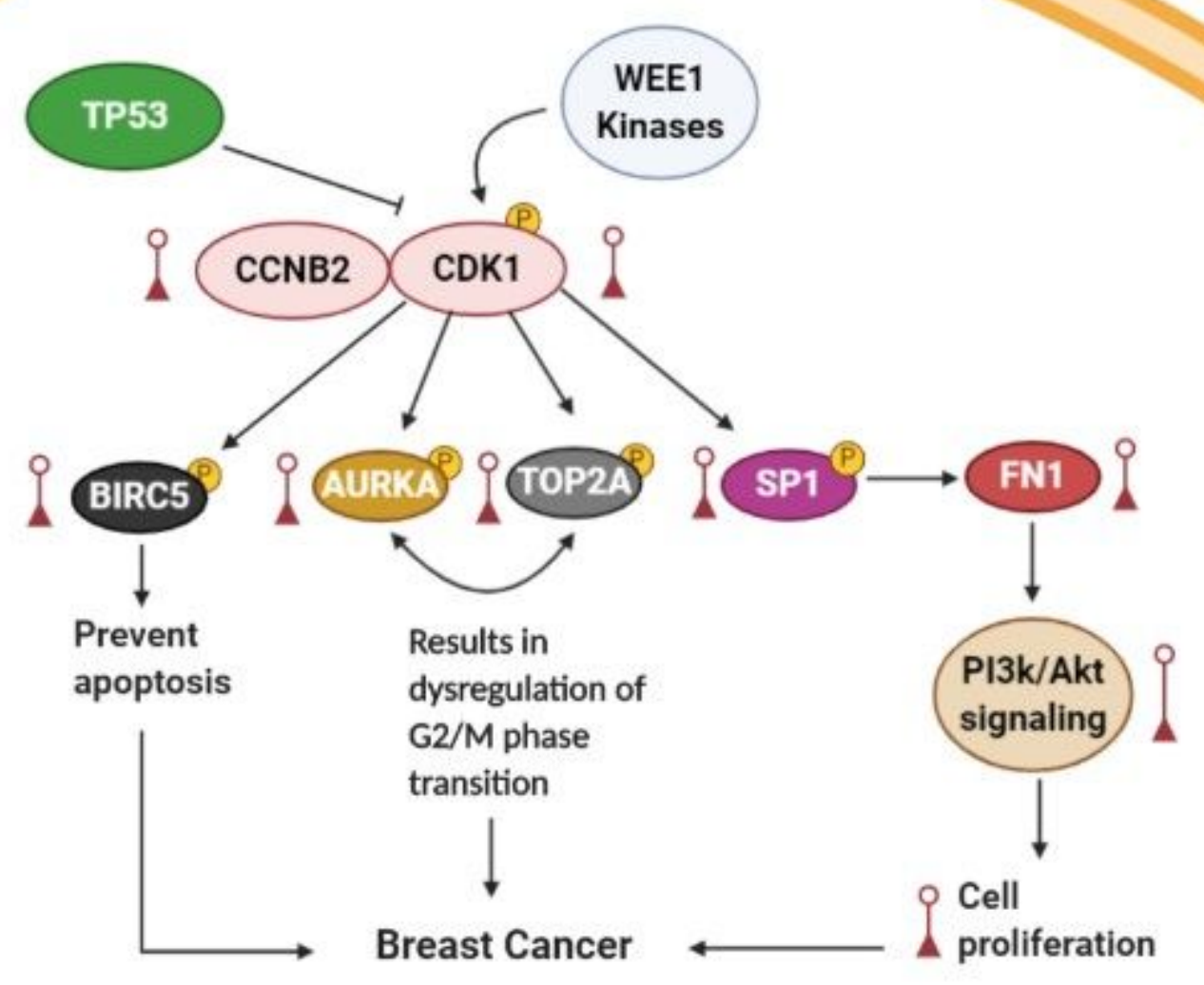

Figure 8

Crosstalk between the hub genes in the identified pathways Cyclin B2 (CCNB2) is a mitotic cyclin that is activated in mitosis and binds to CDK1 to form a complex and thus helps in its activation through phosphorylation by WEE1 kinases. Once CDK1 is activated it further activates BIRC5, AURKA, TOP2A, and SP1 through phosphorylation. After completion of its job, CDK1 is inhibited by TP53. The complex between CCNB2 and CDK1 also known as maturation promoting factor or mitosis promoting factor (MPF) because of its major role in the meiosis process [54]. In the present study, the up-regulation of CDK1 is supposed to elevate the activity of its major target proteins including BIRC5, AURKA, TOP2A, and SP1. The BIRC5 (Survivin) is an anti-apoptosis factor and is normally expressed at a very low level in normal cells [55]. Its high level observed in the present study is supposed to be a major obstacle in the apoptosis of abnormal cells. AURKA and TOP2A both are cell cycle and centrosome cycle regulators which are essential for mitotic onset and cell cycle transition from $G 2$ to $M$ phase [56]. On the basis of our results, it is proposed that overexpressed AURKA and TOP2A increase the G2-M transition and lead to the high proliferation of the cells. SP1 is a zinc finger transcription factor [57] and its elevated expression observed in the current study is expected to up-regulate the expression level of one of its key target gene FN1 which further helps to activate PI3k/Akt signaling pathway and also contributes to the high proliferation of the cells with a different mechanism. In addition, the up-regulation of BIRC5 inhibits the 
apoptosis of such highly proliferative cells and ultimately results in the formation of a tumor. The brown upward arrow show the up-regulation.

\section{Supplementary Files}

This is a list of supplementary files associated with this preprint. Click to download.

- Supplementarydata.docx

- Supplementarydata.docx 